\title{
LAS TABLAS DE MORTALIDAD DE LA POBLACION ANDALUZA
}

\author{
$M .^{a}$ del Pilar ALMOGUERA SALLENT (Coordinadora)* \\ Encarnación AGUILERA TIRADO \\ Mercedes DELGADO MARQUEZ \\ Rafael FLORES CORNEJO \\ Candelaria MIRABENT MARTIN \\ Francisco VICIANA FERNANDEZ
}

\section{INTRODUCCION}

Las tablas de mortalidad viene siendo, desde hace tiempo, una de las técnicas más difundidas y a la vez útiles, a la hora de describir las leyes de la mortalidad de un determinado conjunto demográfico. Según PRESSAT (1967), su cálculo es una de las prácticas más antiguas en demografía, aunque no por ello deja de ser un modelo descriptivo sugestivo; tanto es así, que su uso se ha hecho extensivo a otros sucesos demográficos, aunque eso sí, obteniendo según los casos, resultados desiguales.

Por regla general, una tabla de mortalidad se lleva a cabo a lo largo de análisis longitudinales, esto es, se observa una generación completa desde su nacimiento hasta su total extinción, deduciéndose de dicho análisis las leyes de mortalidad vigentes para tal generación. El estudio resulta absolutamente ajustado a la realidad, pero que duda cabe, se convierte, cuando menos, en una tarea muy laboriosa y de aplicaciones limitadas. Es por ello por lo que el análisis de las condiciones de mortalidad en un momento dado, parece interesar mucho más a los investigadores, ya que esta práctica permite una mayor y más fácil observación de las leyes de mortalidad correspondiente a un grupo demográfico cualquiera, al tiempo que se revela como una labor mucho más util de cara a su aplicación en el seno de las ciencias sociales. Es por ello por lo que el estudio de la mortalidad según un análisis transversal tiene una prioridad evidente.

* Profesora Titular de Geografía Humana. 
La elaboración de las tablas de mortalidad con referencia a un momento «T», se basa en la elección de una generación ficticia a la que se le hace recorrer todas las edades de la vida, sometiéndola en las diferentes edades, a las condiciones de mortalidad observadas en las distintas generaciones reales durante el año (o grupo de años) estudiado. De esta forma resulta fácil comprender, como a través de la aplicación de leyes de mortalidad reales a una generación ficticia, se consigue una magnífica descripción selectiva del comportamiento demográfico por edades frente a la mortalidad; ello de una parte; de otra y al mismo tiempo, permite el cáculo de parámetros tales como la esperanza de vida al nacer, el número de supervivientes a una edad determinada, o el cálculo de probabilidades de supervivencias a edad determinada, tareas todas ellas que se situan en la base o arranque de razonamientos conducentes a la formulación de proyecciones de población y al diseño de modelos de comportamientos demográficos en lo que se refiere a dinámica demográfica.

Incomprensiblemente, la confección de tablas de mortalidad referidas a la población andaluza ha sido una tarea casi marginal en el contexto de los análisis demográficos referidos a la población de nuestra comunidad autónoma, estudios que se han decantado de forma preferente, y nosotros mismo lo hemos hecho así (ALMOGUERA, 1986), por el tratamiento de temas tales como reparto espacial de la población, análisis estructurales de la misma, movimientos migratorios, o estudio de la población activa. Sin embargo y a pesar de ello, es justo reconocer que existen aportaciones sectoriales en las que se han confeccionado tablas de mortalidad para un ámbito espacial concreto de nuestra comunidad autónoma. Tal es el caso del informe de la JUNTA DE ANDALUCIA, 1985, en el que se incluyen tablas de mortalidad para la provincia de Sevilla.

Sin embargo, estudios de conjunto que contengan tablas de mortalidad para toda la población andaluza, y que además hallan sido realizados con cierta perspectiva temporal, no existen hoy por hoy, motivo por si solo que justifica nuestro posterior trabajo. A ello, pues, vamos a dedicar las páginas que siguen.

\section{METODOLOGIA}

Esta investigación fue diseñada como una aproximación preliminar al conocimiento de las diversas funciones biométricas de la población andaluza al objeto de desarrollar, con posterioridad, proyecciones demográficas de ella. En un principio se partió de las estimaciones de las tasas de Mortalidad publicadas por la CONSEJERIA DE SALUD, 1984, de la Junta de Andalucía. A partir de aquí se calcularon las tablas de mortalidad correspondientes a 1979-80 para cada una de las provincias, y sexos. Para ampliar el conocimiento estructural 
(transversal o cross-seccional) de las funciones biométricas suministradas por estas Tablas, se pretendió obtener una visión dinámica de la evolución de ellas calculando dos nuevas visiones estructurales (transversales) de la población andaluza en pasados momentos históricos. La elección de los cortes fue fruto de las transacciones obligadas entre nuestros intereses y las disponibilidades y tabulación de los datos por parte del Instituto Nacional de Estadística. Se partió de la preferencia de escoger períodos censales para optimizar las estimaciones poblacionales. Así, se eligieron censos que dispusieran de datos provinciales al menos en grupos quinquenales; fruto de estas transacciones se terminó por elegir los períodos de 1910-11 y de 1940-41 para realizar los cortes. Por claridad conceptual y disminución del componente aleatorio, se optó por estimar las Tasas a partir de las defunciones del año censal y del inmediatamente siguiente.

La estimación de las tasas promedio de mortalidad para dicho período, se realizó siguiendo la bibliografía consultada ${ }^{1}$ a partir de la división del número de defunciones ocurridas en los períodos bianuales en su grupo de edad específico, partido por la cantidad de personas-tiempo para ese período, estimado este último concepto por la multiplicación de la cantidad de población a mitad de período (en nuestro caso 31-Diciembre) en el grupo de edad específico por longitud del período temporal, (en nuestro caso, dos años), a saber:

Núm. de defunciones a edad $(i, i+5)$

Hab. mitad de período a edad $(i, i+5)$ por long. de período

Las tasas estimadas de esta manera quedan definidas en unidades de defunciones partidas por habitante y año, siendo perfectamente comparables con las más frecuentes estimaciones a partir de datos uni-anuales. Las únicas tasas no estimadas por este método fueron las de menores de un año, para cuyo denominador se emplearon sólo los nacimientos del período.

A partir de estas estimaciones, se calcularon las diversas funciones biométricas de las tablas de mortalidad siguiéndo la metodología recogida por ElandtJohnson (1979) y O.M.S. (1980) y que esquematizamos en el apéndice «A».

Por los abundantes y reiterativos cálculos numéricos, se hizo necesario recurrir a su automatización; se optó por trabajar sobre ordenadores tipo PC y con el Logical de Base de datos DBASEIII, desarrollándose en su lenguaje de programación el programa de cálculo automatizado de las funciones biométricas de las tablas de vida, a partir de las previamente estimadas tasas de mortalidad específicas por edad. Una breve descripción y listado de él se recoge en el apéndice «B».

1. La elaboración de las Tablas de Mortalidad se ha ajustado a los cálculos recomendados por ELANDT-JOHSON, WORLD HIALTH ORGANIZATION. 
No pretendemos extendernos en la descripción pormenorizada de las cuestiones metodológicas, que suelen resultar tediosas incluso para los iniciados. Sin embargo, dada la obligada necesidad de aclarar tales procedimientos en asuntos como el que nos ocupa, es por lo que hemos redactado los apéndices A y $\mathrm{B}$ en los que el lector puede consultar, de manera algo más extensa el proceso mediante el cual hemos establecido nuestros cálculos. Una vez hechas estas observaciones, que creíamos necesarias, damos paso al comentario de las tablas de mortalidad.

\section{MORTALIDAD GENERAL, POR SEXO Y EDADES}

Subrayar la importancia que la mortalidad mantiene como factor explicativo de los cambios demográficos ocurridos tanto en el pasado como en el presente, queda fuera de toda duda. Es por ello por lo que la búsqueda de la obtención de unas «Leyes de Mortalidad» para determinados conjuntos demográficos, es una tarea que parece imponerse en la actualidad en el campo de los estudios demográficos (PUJOL, 1988).

\section{CUADRO I}

TASA BRUTA DE MORTALIDAD

(Fallecidos por 1.000 habitantes y año)

\begin{tabular}{|c|c|c|c|c|c|}
\hline Areas & $1901-10$ & $1921-30$ & $1951-60$ & $1961-70$ & $1971-75$ \\
\hline 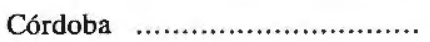 & 27,2 & 20,5 & 8,6 & 8,1 & 8,3 \\
\hline Sevilla & 28,9 & 22,0 & 9,0 & 8,2 & 8,0 \\
\hline 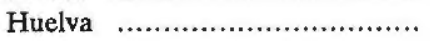 & 23,7 & 18,3 & 10,5 & 10,0 & 9,7 \\
\hline Cádiz $\quad$................................. & 29,2 & 22,9 & 8,8 & 7,5 & 7,3 \\
\hline 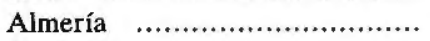 & 28,1 & 20,5 & 9,6 & 8,4 & 8,5 \\
\hline 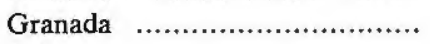 & 27,6 & 19,6 & 8,6 & 8,1 & 8,2 \\
\hline Jaén & 29,7 & 21,9 & 8,9 & 7,9 & 8,2 \\
\hline Málaga & 26,7 & 20,3 & 8,8 & 8,1 & 8,4 \\
\hline ANDALUCIA OC. $\quad . . . \ldots \ldots \ldots \ldots$ & 27,7 & 21,2 & 9,0 & 8,2 & 8,1 \\
\hline ANDALUCIA OR. & 27,8 & 20,6 & 8,9 & 8,1 & 8,3 \\
\hline ANDALUCIA $\quad \ldots \ldots \ldots \ldots \ldots \ldots \ldots$ & 27,8 & 20,9 & 9,0 & 8,2 & 8,2 \\
\hline ........................... & 25,0 & 19,0 & 9,4 & 8,5 & 8,4 \\
\hline
\end{tabular}

Fuente: Anuario Estadístico Andalucía, 1982. 
La mortalidad General en Andalucía, ha ido descendiendo paulatinamente desde comienzos de siglo hasta nuestros días, de tal manera que si bien en ciertos momentos las tasas andaluzas mantuvieron un comportamiento desigual mostrando valores superiores a la media nacional, hoy podemos decir, sin temor a equivocarnos, que sus valores prácticamente se han igualado (vid. Cuadro n. ${ }^{\circ}$ I) salvando, naturalmente, ciertas oscilaciones de las tasas propias de comportamientos coyunturales lógicas e inevitables en el examen de series más o menos largas.

Sin embargo, una aproximación mucho más exacta y elocuente acerca del comportamiento diferencial de la mortalidad, se obtiene calculando su incidencia por grupos de edad y sexo, tareas realizadas para los años establecidos y que pueden consultarse en los Cuadros II y II Bis respectivamente.

El modelo teórico de comportamiento de la mortalidad diferencial según la edad es de todos conocidos: parte de un nivel elevado a 0 años, para decrecer a continuación rapidísimamente alcanzando su valor más bajo en el grupo 1014 años. A partir de esas edades se observa un aumento ininterrumpido de las tasas de mortalidad hasta volver a niveles semejantes a los de la mortalidad infantil en los grupos de edades superiores a los 70-75 años.

Del mismo modo, y refiriéndonos ahora a la mortalidad diferencial según el sexo, ha podidó comprobarse, de manera empírica, una mortalidad masculina superior a la femenina en todas las edades; se trata del fenómeno denominado supermortalidad masculina, circunstancia que resulta tan conocida, que casi no necesita explicación.

Pues bien, vamos a analizar, seguidamente, hasta qué punto las líneas correspondientes a la mortalidad diferencial obtenidas para Andalucía en los años estudiados, se ajustan o no al modelo teórico establecido.

Como puede observarse (vid. gráfico $n .^{\circ} 1$ y 1 bis) las líneas que representan la mortalidad a comienzos de siglo tanto para Andalucía como para el conjunto del País, dibujan el perfil teórico ya descrito, esto es, altos valores en mortalidad infantil (0-1 años) para descender bruscamente hasta alcanzar un mínimo en el grupo 15-19. A partir de esas edades las tasas se mantienen casi estacionarias, volviéndose a producir una nueva inflexión en torno a los 25-29 años, edad que señala el punto de recuperación de la curva de mortalidad, que seguirá en ascenso hasta alcanzar los más altos valores en los grupos de edades que representan a una misma generación.

Sin embargo, y aunque ello sea cierto, hemos de realizar algunas matizaciones a nuestro modo de ver bastantes significativas.

Así, y en lo que se refiere a la mortalidad correspondente a 1910 puede observarse que Andalucía y España mantenían un mismo comportamiento diferencial de la mortalidad en el grupo masculino caracterizado por: 


\section{CUADRO II.a}

TASAS DE MORTALIDAD $\left({ }^{*} 1.000\right.$ HAB. $)$. MUJERES ANDALUCIA TOTAL Y PROVINCIAS. ESPAÑA TOTAL

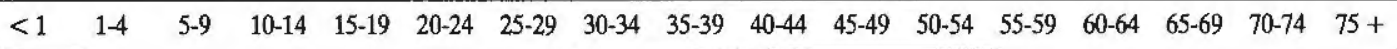

1910-11

\begin{tabular}{|c|c|c|c|c|c|c|c|c|c|c|c|c|c|c|c|c|c|}
\hline Almería & 156,1 & 58,9 & 7,6 & 3,3 & 5,8 & 8,4 & 7,4 & 7,6 & 9,8 & 8,7 & 10,2 & 13,2 & 17,9 & 35,4 & 47,3 & 99,8 & 265,8 \\
\hline 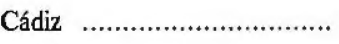 & 169,3 & 48,2 & 8,5 & 3,6 & 6,3 & 9,5 & 9,8 & 9,3 & 9,4 & 12,1 & 9,6 & 14,8 & 20,7 & 33,5 & 50,5 & 86,9 & 199,9 \\
\hline 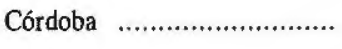 & 154,5 & 51,7 & 7,5 & 2,6 & 5,2 & 6,9 & 7,6 & 8,3 & 8,6 & 7,9 & 8,7 & 10,0 & 19,1 & 32,2 & 55,4 & 89,7 & 205,8 \\
\hline Granada & 160,9 & 59,7 & 6,9 & 3,2 & 7,2 & 8,2 & 7,1 & 9,4 & 10,8 & 8,9 & 8,9 & 12,5 & 16,0 & 32,7 & 50,7 & 98,9 & 208,7 \\
\hline Huelva & 141,5 & 33,7 & 5,7 & 2,7 & 5,2 & 7,0 & 7,6 & 8,1 & 7,1 & 8,7 & 7,9 & 11,2 & 16,3 & 25,3 & 40,8 & 81,0 & 194,3 \\
\hline Jaén & 199,3 & 48,8 & 7,4 & 4,0 & 6,5 & 7,0 & 9,2 & 11,1 & 10,0 & 12,1 & 11,0 & 18,3 & 29,4 & 27,1 & 38,2 & 120,2 & 147,6 \\
\hline Málaga & 160,5 & 51,2 & 6,0 & 5,2 & 5,2 & 8,4 & 9,2 & 8,7 & 10,1 & 9,1 & 19,8 & 16,4 & 19,4 & 25,0 & 46,5 & 87,9 & 222,4 \\
\hline Sevilla & 172,8 & 53,7 & 7,4 & 3,7 & 6,3 & 8,8 & 8,9 & 8,6 & 10,2 & 10,5 & 7,3 & 10,7 & 18,7 & 27,6 & 46,6 & 80,4 & 189,9 \\
\hline TOTAL ANDALUCIA ....... & 166,6 & 51,6 & 7,2 & 3,6 & 6,0 & 8,1 & 8,4 & 9,0 & 9,6 & 9,8 & 10,4 & 13,4 & 19,6 & 29,8 & 47,1 & 91,3 & 202,4 \\
\hline ESPAN̈A & 146,5 & 43,9 & 6,7 & 3,5 & 5,7 & 7,2 & 7,8 & 8,4 & 9,1 & 9,6 & 10,7 & 14,1 & 19,6 & 32,3 & 50,5 & 89,6 & 164,3 \\
\hline
\end{tabular}


CUADRO II.a (Continuación)

TASAS DE MORTALIDAD (*1.000 HAB.). MUJERES

ANDALUCIA TOTAL Y PROVINCIAS. ESPAÑA TOTAL

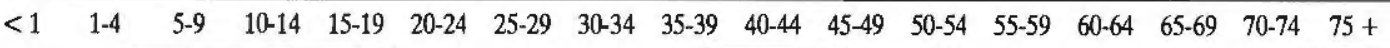

\begin{tabular}{|c|c|c|c|c|c|c|c|c|c|c|c|c|c|c|c|c|c|}
\hline & $<1$ & $1-4$ & $5-9$ & $10-14$ & $15-19$ & $20-24$ & $25-29$ & $30-34$ & $35-39$ & $40-44$ & $45-49$ & $50-54$ & $55-59$ & $60-64$ & $65-69$ & $70-74$ & $75+$ \\
\hline \multicolumn{18}{|c|}{$1940-41$} \\
\hline Almería & 128,4 & 19,9 & 3,5 & 2,8 & 4,0 & 4,7 & 5,2 & 5,6 & 6,2 & 7,2 & 9,6 & 15,4 & 15,4 & 28,5 & 36,2 & 87,9 & 186,4 \\
\hline 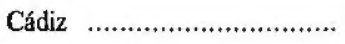 & 136,9 & 17,4 & 3,1 & 2,5 & 4,3 & 5,6 & 6,0 & 5,8 & 7,9 & 7,9 & 10,5 & 13,4 & 20,4 & 32,6 & 50,3 & 92,4 & 201,5 \\
\hline Córdoba ............................... & 77,0 & 11,4 & 1,9 & 1,2 & 1,8 & 2,3 & 2,6 & 2,4 & 3,0 & 3,1 & 3,8 & 5,4 & 7,1 & 11,6 & 17,6 & 30,9 & 77,3 \\
\hline 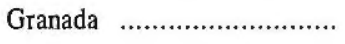 & 71,8 & 10,7 & 1,8 & 1,1 & 1,7 & 2,0 & 2,5 & 2,7 & 3,5 & 3,8 & 4,3 & 5,9 & 8,4 & 14,6 & 18,5 & 40,2 & 93,6 \\
\hline Huelva & 58,3 & 7,2 & 1,4 & 1,4 & 1,5 & 2,4 & 2,2 & 2,9 & 2,8 & 2,4 & 3,2 & 5,7 & 5,5 & 9,1 & 15,3 & 26,7 & 75,6 \\
\hline Jaén & 94,2 & 14,5 & 2,4 & 1,1 & 1,9 & 2,7 & 3,4 & 3,2 & 3,3 & 4,2 & 4,9 & 6,4 & 8,7 & 14,5 & 21,8 & 41,5 & 94,8 \\
\hline 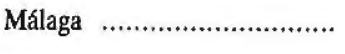 & 57,8 & 8,0 & 1,5 & 1,1 & 1,5 & 2,1 & 2,8 & 2,6 & 2,9 & 3,3 & 3,5 & 5,0 & 6,4 & 11,7 & 17,7 & 28,3 & 83,4 \\
\hline Sevilla & 72,2 & 8,5 & 1,8 & 1,0 & 1,9 & 2,0 & 2,1 & 2,9 & 2,8 & 3,1 & 3,6 & 5,6 & 7,1 & 11,8 & 16,8 & 31,3 & 76,7 \\
\hline TOTAL ANDALUCIA $\ldots . . .$. & 85,5 & 11,9 & 2,1 & 1,4 & 2,2 & 2,8 & 3,1 & 3,3 & 3,8 & 4,1 & 5,1 & 7,2 & 9,3 & 15,9 & 23,1 & 44,7 & 106,1 \\
\hline ESPAÑA & 116,6 & 15,5 & 3,4 & 2,3 & 3,4 & 4,4 & 4,8 & 5,2 & 5,8 & 6,6 & 8,3 & 11,1 & 15,9 & 24,5 & 39,0 & 67,7 & 251,1 \\
\hline
\end{tabular}


CUADRO II.a (Conclusión)

TASAS DE MORTALIDAD (*1.000 HAB.). MUJERES

ANDALUCIA TOTAL Y PROVINCIAS. ESPAÑA TOTAL

\begin{tabular}{lllllllllllllllll}
$<1$ & $1-4$ & $5-9$ & $10-14$ & $15-19$ & $20-24$ & $25-29$ & $30-34$ & $35-39$ & $40-44$ & $45-49$ & $50-54$ & $55-59$ & $60-64$ & $65-69$ & $70-74$ & $75+$ \\
\hline
\end{tabular}

1975-79

\begin{tabular}{|c|c|c|c|c|c|c|c|c|c|c|c|c|c|c|c|c|c|}
\hline Almería & 18,5 & 0,6 & 0,3 & 0,2 & 0,3 & 0,2 & 0,6 & 0,8 & 1,1 & 1,4 & 2,0 & 3,8 & 5,3 & 8,8 & 20,1 & 29,9 & 104,9 \\
\hline Cádiz & 14,7 & 0,8 & 0,3 & 0,2 & 0,3 & 0,3 & 0,4 & 0,8 & 1,0 & 1,7 & 3,0 & 4,4 & 6,4 & 11,2 & 20,4 & 33,3 & 102,7 \\
\hline 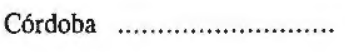 & 29,7 & 0,6 & 0,2 & 0,3 & 0,2 & 0,4 & 0,5 & 0,6 & 0,8 & 1,4 & 2,1 & 3,5 & 5,0 & 9,3 & 15,3 & 29,1 & 89,0 \\
\hline Granada $\ldots \ldots \ldots \ldots \ldots \ldots \ldots \ldots \ldots$ & 18,4 & 0,7 & 0,3 & 0,2 & 0,3 & 0,4 & 0,6 & 0,7 & 0,9 & 1,7 & 2,5 & 3,9 & 5,5 & 9,2 & 16,9 & 31,1 & 99,1 \\
\hline Huelva & 17,2 & 0,6 & 0,2 & 0,3 & 0,3 & 0,3 & 0,5 & 0,7 & 1,3 & 1,4 & 3,1 & 3,6 & 5,7 & 8,8 & 18,2 & 34,0 & 101,1 \\
\hline Jaén & 16,9 & 0,5 & 0,2 & 0,2 & 0,3 & 0,3 & 0,5 & 0,6 & 0,9 & 1,4 & 2,5 & 3,3 & 5,0 & 8,6 & 16,1 & 30,4 & 93,3 \\
\hline 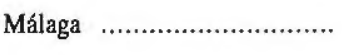 & 15,6 & 0,6 & 0,3 & 0,2 & 0,3 & 0,4 & 0,6 & 0,8 & 0,8 & 1,7 & 2,7 & 3,6 & 6,4 & 10,5 & 16,6 & 32,2 & 98,1 \\
\hline 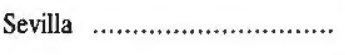 & 12,8 & 0,6 & 0,3 & 0,2 & 0,3 & 0,4 & 0,4 & 0,8 & 0,8 & 1,8 & 2,6 & 3,8 & 6,0 & 10,1 & 15,8 & 32,9 & 93,5 \\
\hline TOTAL ANDALUCIA ...... & 16,3 & 0,6 & 0,3 & 0,2 & 0,3 & 0,4 & 0,5 & 0,8 & 0,9 & 1,6 & 2,6 & 3,9 & 5,7 & 9,8 & 17,0 & 31,7 & 96,7 \\
\hline ESPAÑA & 14,2 & 0,7 & 0,3 & 0,3 & 0,3 & 0,4 & 0,5 & 0,7 & 0,9 & 1,6 & 2,4 & 3,8 & 5,5 & 9,1 & 15,8 & 29,0 & 89,7 \\
\hline
\end{tabular}




\section{CUADRO II.b}

TASAS DE MORTALIDAD (*1.000 HAB.). VARONES

ANDALUCIA TOTAL Y PROVINCIAS. ESPAÑA TOTAL

\begin{tabular}{|c|c|c|c|c|c|c|c|c|c|c|c|c|c|c|c|c|c|}
\hline & $<1$ & $1-4$ & $5-9$ & $10-14$ & $15-19$ & $20-24$ & $25-29$ & $30-34$ & $35-39$ & $40-44$ & $45-49$ & $50-54$ & $55-59$ & $60-64$ & $65-69$ & $70-74$ & $75+$ \\
\hline \multicolumn{18}{|c|}{$1910-11$} \\
\hline 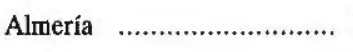 & 164,2 & 59,2 & 8,3 & 2,9 & 7,1 & 8,0 & 7,8 & 10,2 & 11,4 & 12,2 & 17,4 & 19,3 & 27,7 & 39,0 & 61,9 & 103,9 & 245,1 \\
\hline 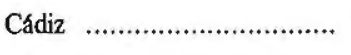 & 185,1 & 50,5 & 7,8 & 3,2 & 6,4 & 6,4 & 7,5 & 9,3 & 11,2 & 14,1 & 17,6 & 21,4 & 34,8 & 49,0 & 78,2 & 109,1 & 223,5 \\
\hline 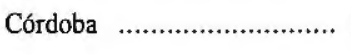 & 171,4 & 55,7 & 6,9 & 2,4 & 5,6 & 6,5 & 5,8 & 6,8 & 8,1 & 9,7 & 11,1 & 14,7 & 23,4 & 40,1 & 72,2 & 99,8 & 194,8 \\
\hline Granada & 174,9 & 56,8 & 6,9 & 3,7 & 4,5 & 6,6 & 6,6 & 7,1 & 9,2 & 13,7 & 12,1 & 16,5 & 26,5 & 41,9 & 58,5 & 95,9 & 219,1 \\
\hline Huelva & 158,9 & 34,9 & 4,8 & 3,0 & 4,3 & 7,0 & 7,9 & 7,6 & 9,8 & 11,9 & 11,9 & 15,4 & 30,1 & 41,4 & 61,0 & 107,5 & 222,7 \\
\hline Jaén & 192,1 & 53,9 & 7,3 & 3,9 & 5,4 & 6,9 & 7,9 & 8,3 & 10,2 & 11,5 & 9,9 & 15,6 & 23,6 & 37,6 & 57,0 & 133,2 & 214,8 \\
\hline Málaga & 165,8 & 53,2 & 6,0 & 3,2 & 5,2 & 8,0 & 6,9 & 7,7 & 9,5 & 10,4 & 31,1 & 21,1 & 28,4 & 34,7 & 65,5 & 96,5 & 235,6 \\
\hline Sevilla $\ldots \ldots \ldots \ldots \ldots \ldots \ldots \ldots \ldots \ldots$ & 194,6 & 55,0 & 7,3 & 3,3 & 5,7 & 8,6 & 7,8 & 8,2 & 10,7 & 13,7 & 9,2 & 17,3 & 29,3 & 41,2 & 60,5 & 96,0 & 211,1 \\
\hline TOTAL ANDALUCIA ...... & 177,5 & 53,4 & 7,0 & 3,2 & 5,5 & 7,2 & 7,2 & 8,1 & 10,0 & 12,1 & 14,6 & 17,6 & 27,7 & 40,5 & 64,1 & 102,7 & 219,2 \\
\hline ESPAN̈A & 163,9 & 46,1 & 6,6 & 3,2 & 5,6 & 7,4 & 7,2 & 7,7 & 9,2 & 10,7 & 13,8 & 17,6 & 25,6 & 37,9 & 59,2 & 94,0 & 134,5 \\
\hline
\end{tabular}




\section{CUADRO II.b (Continuación)}

TASAS DE MORTALIDAD $\left({ }^{*} 1.000\right.$ HAB.). VARONES ANDALUCIA TOTAL Y PROVINCIAS. ESPAÑA TOTAL

\begin{tabular}{|c|c|c|c|c|c|c|c|c|c|c|c|c|c|c|c|c|c|}
\hline & $<1$ & $1-4$ & $5-9$ & $10-14$ & $15-19$ & $20-24$ & $25-29$ & $30-34$ & $35-39$ & $40-44$ & $45-49$ & $50-54$ & $55-59$ & $60-64$ & $65-69$ & $70-74$ & $75+$ \\
\hline \multicolumn{18}{|c|}{$1940-41$} \\
\hline Almería & 147,8 & 20,4 & 4,1 & 2,3 & 4,7 & 9,3 & 11,6 & 10,1 & 12,3 & 16,1 & 19,7 & 25,0 & 38,0 & 47,9 & 74,6 & 110,6 & 291,5 \\
\hline Cádiz & 156,6 & 17,0 & 3,9 & 2,8 & 5,1 & 10,2 & 11,6 & 10,0 & 13,6 & 18,6 & 29,3 & 36,5 & 53,0 & 77,9 & 101,9 & 145,5 & 268,4 \\
\hline Córdoba & 84,1 & 10,4 & 2,0 & 1,5 & 2,2 & 4,5 & 7,2 & 6,0 & 6,5 & 7,1 & 9,4 & 12,2 & 14,1 & 22,5 & 32,5 & 46,9 & 102,0 \\
\hline Granada ............................ & 81,1 & 10,7 & 1,7 & 1,4 & 2,2 & 3,7 & 4,5 & 4,6 & 5,0 & 6,1 & 8,4 & 9,6 & 14,6 & 22,9 & 35,0 & 52,3 & 101,9 \\
\hline 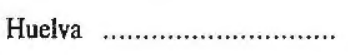 & 58,8 & 6,3 & 1,8 & 1,2 & 1,8 & 3,6 & 4,7 & 4,3 & 5,0 & 6,9 & 9,6 & 12,0 & 16,7 & 23,1 & 30,2 & 43,2 & 82,3 \\
\hline Jaén & 108,5 & 14,9 & 2,6 & 1,4 & 2,8 & 6,1 & 7,6 & 7,9 & 8,4 & 9,5 & 11,3 & 13,9 & 19,0 & 26,7 & 37,3 & 60,5 & 125,5 \\
\hline Málaga ............................ & 68,8 & 7,1 & 1,9 & 1,1 & 1,9 & 3,9 & 4,7 & 5,7 & 5,7 & 6,0 & 8,7 & 9,9 & 12,7 & 19,0 & 25,1 & 44,5 & 98,5 \\
\hline Sevilla & 77,2 & 8,9 & 1,9 & 1,4 & 2,4 & 3,0 & 3,7 & 3,7 & 4,7 & 6,9 & 9,2 & 11,3 & 15,4 & 20,2 & 28,0 & 45,9 & 102,1 \\
\hline TOTAL ANDALUCIA ...... & 96,1 & 11,8 & 2,4 & 1,6 & 2,7 & 5,3 & 6,6 & 6,2 & 7,2 & 9,0 & 12,4 & 15,1 & 20,9 & 29,8 & 42,3 & 64,2 & 135,5 \\
\hline ESPAN̈A & 115,8 & 16,4 & 4,0 & 2,8 & 5,4 & 10,6 & 12,1 & 12,2 & 13,2 & 14,9 & 20,0 & 24,9 & 35,6 & 47,4 & 71,6 & 106,8 & 190,6 \\
\hline
\end{tabular}




\section{CUADRO II.b (Conclusión)}

TASAS DE MORTALIDAD (*1.000 HAB.). VARONES

ANDALUCIA TOTAL Y PROVINCIAS. ESPAÑA TOTAL

\begin{tabular}{|c|c|c|c|c|c|c|c|c|c|c|c|c|c|c|c|c|c|}
\hline & $<1$ & $1-4$ & $5-9$ & $10-14$ & $15-19$ & $20-24$ & $25-29$ & $30-34$ & $35-39$ & $40-44$ & $45-49$ & $50-54$ & $55-59$ & $60-64$ & $65-69$ & $70-74$ & $75+$ \\
\hline \multicolumn{18}{|c|}{$1975-79$} \\
\hline Almería & 20,7 & 0,9 & 0,4 & 0,4 & 1,0 & 1,2 & 1,5 & 1,4 & 1,5 & 3,4 & 4,5 & 7,8 & 11,8 & 18,7 & 32,3 & 51,6 & 121,1 \\
\hline Cádiz & 21,2 & 1,0 & 0,4 & 0,3 & 0,7 & 1,1 & 1,1 & 1,6 & 2,0 & 3,8 & 5,6 & 10,0 & 14,2 & 24,8 & 43,0 & 66,1 & 128,9 \\
\hline Córdoba & 23,6 & 0,6 & 0,3 & 0,3 & 0,7 & 0,8 & 0,9 & 1,6 & 1,5 & 2,8 & 4,4 & 7,1 & 10,2 & 17,8 & 31,5 & 50,5 & 114,4 \\
\hline Granada & 21,9 & 0,8 & 0,3 & 0,3 & 0,7 & 0,9 & 1,0 & 1,3 & 1,8 & 3,1 & 3,8 & 7,3 & 10,2 & 20,1 & 32,5 & 53,1 & 111,0 \\
\hline Huelva & 22,3 & 0,8 & 0,4 & 0,3 & 0,7 & 1,0 & 1,1 & 1,6 & 1,7 & 3,4 & 5,4 & 8,4 & 13,1 & 19,9 & 40,7 & 66,7 & 134,2 \\
\hline Jaén & 23,1 & 0,7 & 0,4 & 0,4 & 0,7 & 0,8 & 1,0 & 1,3 & 1,5 & 2,5 & 4,4 & 6,7 & 10,2 & 16,4 & 30,6 & 47,2 & 109,6 \\
\hline Málaga .................................... & 20,7 & 0,7 & 0,3 & 0,3 & 0,8 & 1,1 & 1,2 & 1,4 & 1,9 & 3,5 & 5,6 & 9,6 & 13,9 & 22,2 & 35,8 & 57,5 & 113,0 \\
\hline Sevilla & 16,9 & 0,9 & 0,4 & 0,3 & 0,7 & 0,9 & 0,9 & 1,2 & 1,6 & 3,4 & 5,7 & 9,4 & 13,6 & 20,9 & 35,5 & 54,8 & 119,8 \\
\hline TOTAL ANDALUCIA ...... & 20,5 & 0,8 & 0,4 & 0,3 & 0,7 & 0,9 & 1,1 & 1,4 & 1,7 & 3,2 & 5,0 & 8,5 & 12,3 & 20,4 & 35,2 & 55,4 & 117,5 \\
\hline ESPAÑA & 18,0 & 0,9 & 0,4 & 0,4 & 0,9 & 1,1 & 1,2 & 1,4 & 1,7 & 3,2 & 4,8 & 8,0 & 11,4 & 18,4 & 31,0 & 49,7 & 109,0 \\
\hline
\end{tabular}



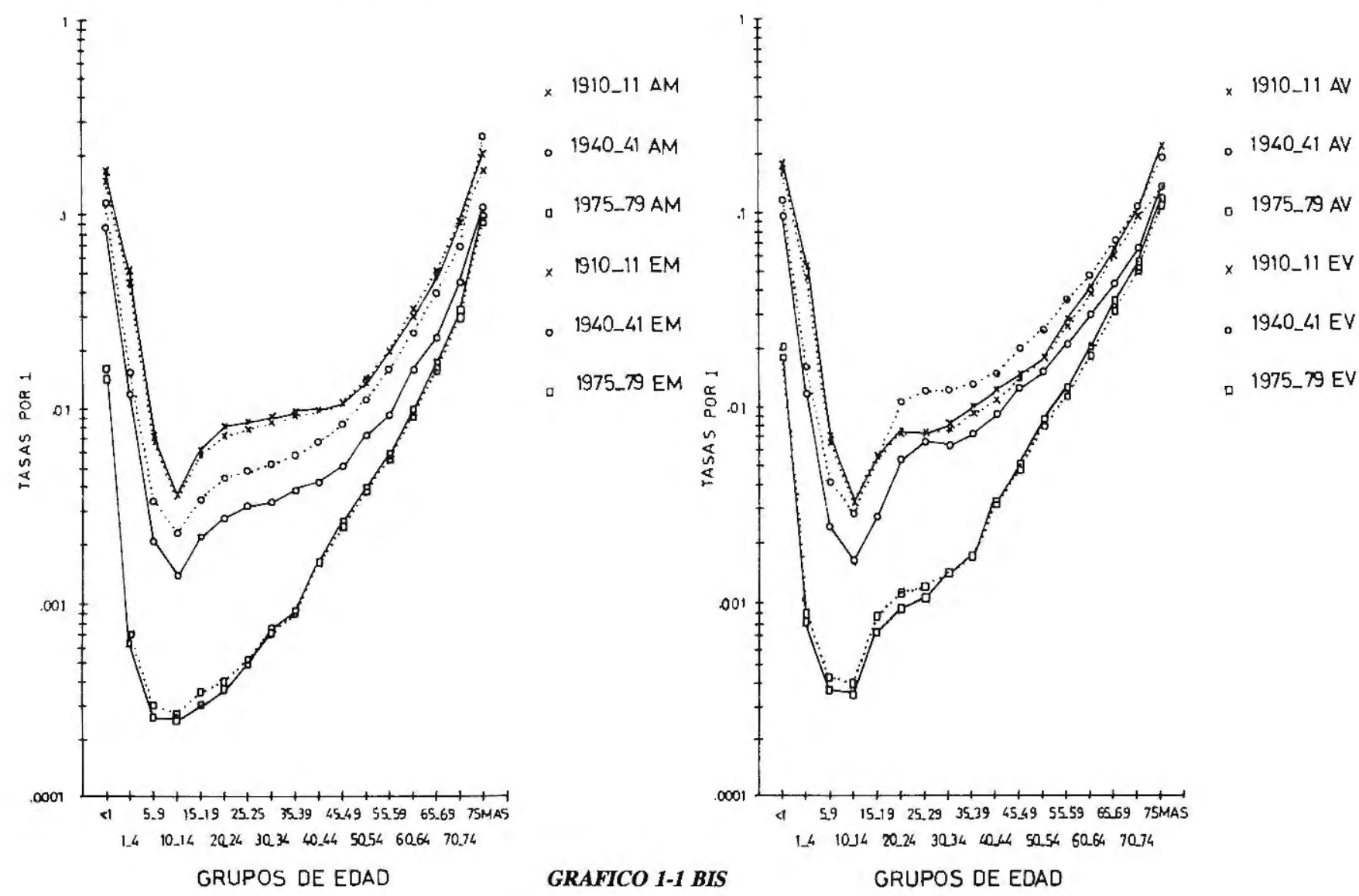
GRUPOS DE EDAD 
a) Fortísima mortalidad infantil, alcanzando valores del orden del $177 \%$, hecho que expresa, por sí mismo, cuales eran las condiciones socio-económicas imperantes en nuestro país. No olvidemos que la mortalidad infantil viene utilizándose, de forma generalizada, como uno de los indicadores básicos del desarrollo económico y sanitario de las diferentes regiones. Destaquemos únicamente como salvedad, que la mortalidad infantil era aun más alta en el caso de Andalucía.

b) A partir del grupo 1-4 las líneas que representan las distintas mortalidades por grupos de edades, sou prácticamente coincidentes, destacando tan sólo la obtención de tasas ligeramente más elevadas para la población andaluza. De todas formas conviene destacar que las tasas obtenidas para los grupos adultos jóvenes y adultos maduros son elevadas con respecto a lo que suele ser la norma general.

c) Los valores de las tasas de los últimos grupos generacionales, alcanzaban valores semejantes a los registrados para la mortalidad infantil en los mismos años.

d) El fenómeno de la supermortalidad masculina se observaba claramente en los cocientes referidos a la mortalidad infantil, y a partir del grupo 30-34 años.

En definitiva y como conclusión puede afirmarse que, en 1910 la mortalidad diferencial por sexo y edad correspondiente a Andalucía mantenía las mismas características que las obtenidas para el conjunto nacional, aunque con valores algo más elevados. Naturalmente ello nos obliga a considerar que para ambos grupos demográficos, las leyes de mortalidad eran semejantes, sieudo las propias que definen a un régimen demográfico antiguo en el que la selección natural por mortalidad en todas las edades era la tónica general.

Si acudimos ahora al examen del comportamiento de la mortalidad para los años 1940-41, podemos observar (vid. Cuadros II y II Bis), que las trayectorias de las líneas de mortalidad andaluza y española tanto por grupos de edades como según el sexo, eran prácticamente semejantes a las descritas para 1910 ajustándose, por tanto, al mismo modelo teórico ya señalado. Ahora bien, pese a ello, cabe destacar:

a) Puede observarse un descenso generalizado de la mortalidad andaluza en todas las edades, descenso que se manifiesta tanto para el grupo masculino como para el femenino. Esta disminución, que se materializa en unas líneas que aparecen siempre representadas por debajo de las correspondientes a 1910 , resulta especialmente significativa en el caso de la mortalidad infantil. 
b) El modelo teórico descrito se interrumpe bruscamente por el espectacular ascenso originado en las tasas de mortalidad masculina para el conjunto nacional; y tal es la desviación que se produce en el modelo teórico que venimos comentando, que la línea de mortalidad supera con creces la correspondiente a 1910 , por lo que podemos calificarla perfectamente como de supermortalidad catastrófica (si nos atenemos, naturalmente, a las causas que la provocaron). La mortalidad más alta del siglo, pues, ateniéndonos a los cortes temporales realizados, se produjo en estas fechas. No obstante ello no se cumple para la población infantil, que mantiene su mortalidad a la baja. Pensamos, no obstante, que este descenso de la mortalidad infantil se debe más a un efecto estadístico que no a un descenso real (disminución brusca de los nacimientos).

Este comportamiento que acabamos de describir nos induce a pensar que la población andaluza pudo hacer frente de forma mucho más eficaz el fenómeno de la supermortalidad correspondiente a la guerra civil, y ello, bien por tratarse de una población profundamente rural, bien por poseer unos efectivos en su conjunto sensiblemente más jóvenes que los correspondientes al conjunto nacional.

En definitiva, la mortalidad diferencial según sexo y edad para 1940-41, registraba un modelo de comportmaiento a la baja pero caracterizado aún por la existencia de niveles considerables de mortalidad en todos los grupos de edades. Ello viene a señalar, que duda cabe, la existencia aún de sucesos fatales de naturaleza exógena y que, por tanto, afectaban diferencialmente a todas las edades. El modelo, a nuestro juicio, no puede calificarse aun de «transicional»; creemos estar todavía ante la presencia de un régimen antiguo alterado por los sucesos políticos y sociales de todos conocidos.

Para 1975-79, último de los cortes temporales realizados ${ }^{2}$, podemos decir, ahora sí, que hemos observado importantes modificaciones en el modelo teórico que hasta ahora venimos describiendo.

Por lo que se refiere a las curvas masculinas, cabe destacar, efectivamente, que el perfil de las líneas se mantienen semejantes las correspondientes a los años anteriores en el caso de las dos inflexiones más importantes, esto es, el momento de la transición de los grupos «jóvenes» a los «adultos maduros», de otra. Sin embargo, la modificación más evidente en las líneas de las trayectorias se sitúa en:

2. Nos ha resultado prácticamente imposible realizar un corte temporal más actualizado, debido a la falta de estadísticas lo suficientemente desagregadas como para permitir el cálculo de una tabla de mortalidad más próxima en el tiempo. 
a) El arranque de las mismas, mucho más bajas que las anteriores, fenómeno imputable, lógicamente, a una más débil mortalidad infantil, siendo para Andalucía del $18 \%$. Si tenemos en cuenta que dicho parámetro en 1910 era del $177 \%$, bien podemos afirmar que el descenso de la mortalidad infantil, tanto en España como en Analucía en lo que va de siglo ha sido uno de los triunfos más evidentes en la lucha del liombre contra la mortalidad.

b) La segunda gran innovación se produce en las edades medias, de tal forma que entre los grupos 1-4 hasta el 33-39 ambos inclusive, la mortalidad era prácticamente insignificante dado los bajísimos valores obtenidos, que en la mayoría de los casos ni siquiera llegaba a alcanzar el $1 \%$, este descenso la sido especialmente espectacular en el grupo 1-4 años. Esta situación se explica nuevamente por la naturaleza de las defunciones; la casi total desaparición de la mortalidad exógena, hace que las defunciones se produzcan principalmente por sucesos endógenos, lo que tiende a concentrar la mortalidad en los grupos superiores de cada generación.

c) La tercera innovación radica pues, en la no coincidencia de los umbrales de comienzo y fin de la mortalidad diferencial según generaciones, ya que la mortalidad en el grupo anciano adquiere, ahora sí, unos valores muy superiores a los correspondientes a la mortalidad infantil.

En consecuencia, puede afirmarse con todo rigor, que nos encontramos con un modelo de comportamiento respecto a la mortalidad, claramente distinto de los anteriores, modelo que bien podemos calificar de «evolucionado», al menos en lo que a las leyes de mortalidad se refiere.

Cabe ahora preguntarse si este nuevo modelo que acabamos de describirse ajusta y explica la mortalidad diferencial para Andalucía en estas fechas; y la respuesta es sorprendente: se ajusta, pero manteniendo tasas manifiestamente más bajas en las edades correspondientes a los grupos jóvenes y adulto-jóvenes, siendo en el grupo de anciano la mortalidad superior. Por tanto, cuando se afirma —como viene siendo frecuente en los análisis basados sólo en las oscilaciones de las tasas brutas - que la mortalidad andaluza es superior al conjunto nacional, se está cometiendo, cuando menos, una importante inexactitud que es necesario corregir; al menos ello es lo que se deduce de la mortalidad diferencial según edades.

El modelo femenino para los años estudiados presenta los mismos caracteres que ya de forma reiterada venimos describiendo. Sin embargo, y siendo válidas todas las afirmaciones que acabamos de realizar, es necesario añadir una más, que es, para el caso femenino, la supresión del cambio de tendencia 
que de una manera brusca se produce en torno al grupo 15-19 años y que señala un aumento brusco de la mortalidad al pasar de la edad joven a la adultajoven. La causa de la supresión de tal comportamiento puede explicarse, y lo esbozamos como hipótesis, en una modificación en las leyes de la fecundidad, así como por una mejor asistencia sanitaria que ha venido a evidenciar la mayor resistencia biológica de la mujer frente a la muerte.

\section{ANALISIS DE LA ESPERANZA DE VIDA}

La elaboración de las Tablas de Mortalidad permiten, además de un examen detenido de la mortalidad diferencial según sexo y grupos de edades, la obtención de un segundo parámetro de gran valor a la hora de determinar los comportamientos naturales de un conjunto demográfico cualquiera. Nos estamos refiriendo, naturalmente, a los cálculos de la Esperanza de Vida.

La esperanza de vida tiene por objeto averiguar cuál es la edad media de las personas fallecidas a lo largo de una generación, o lo que es lo mismo, calcular la duración media de su vida. Ahora bien, si en vez de llevar a cabo un análisis de naturaleza longitudinal, lo efectuamos desde un punto de vista transversal o en el momento «T», el concepto se mantiene inalterable, es decir, se trata de averiguar cuál es la duración media de la vida para todo un conjunto demográfico y en un período concreto. Esto es lo que hemos realizado para la población andaluza siguiendo los cortes temporales ya anteriormente descritos. Para mayor comodidad en su comentario hemos elaborado los cuadros III y III bis así como los gráficos $n .^{\circ} 2$ y 2 bis. En estos últimos aparecen dibujadas las líneas que nos indican la esperanza de vida de la población andaluza según el sexo. Las gráficas, en este caso, permiten una lectura mucho más comoda.

Como puede observarse, para 1910 la esperanza de vida en general podía calificarse de muy baja tanto para Andalucía como para el conjunto nacional, y aunque ello era así para todas las edades, resultaba especialmente significativa la esperanza de vida al nacer ( 0 años), que tan sólo registraba un horizonte de 39 años para los andaluces y 41 años para los españoles. Esta bajísima esperanza de vida al nacer reflejaba, de modo elocuente, las pésimas condiciones de vida imperantes en la España de principios de siglo, situación plenamente compartida por Andalucía, que llegaba a manifestar una esperanza de vida aún más baja si cabe que la correspondiente al conjunto del país.

Las diferencias en las expectativas de vida según los sexos y para los mismos años, manifestaban una mayor esperanza para el sexo femenino, conjunto que registraba esperanzas de vida superiores a la de los hombres en todas las edades, este hecho viene a reiterar la idea, ya antes señalada y aceptada de forma 
CUADRO III.a

ESPERANZA DE VIDA. MUJERES

ANDALUCIA TOTAL Y PROVINCIAS. ESPAÑA TOTAL

\begin{tabular}{|c|c|c|c|c|c|c|c|c|c|c|c|c|c|c|c|c|}
\hline & 0 & 1 & 5 & 10 & 15 & 20 & 25 & 30 & 35 & 40 & 45 & 50 & 55 & 60 & 65 & 70 \\
\hline \multicolumn{17}{|c|}{$1910-11$} \\
\hline Almería & 40,22 & 45,59 & 53,01 & 49,98 & 45,77 & 42,04 & 38,73 & 35,10 & 31,36 & 27,80 & 23,91 & 20,03 & 16,22 & 12,50 & 9,43 & 6,27 \\
\hline 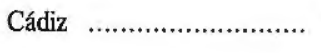 & 40,47 & 46,42 & 51,77 & 48,91 & 44,76 & 41,09 & 37,97 & 34,76 & 31,29 & 27,67 & 24,23 & 20,29 & 16,65 & 13,19 & 10,14 & 7,33 \\
\hline Córdoba & 42,23 & 47,84 & 54,26 & 51,25 & 46,89 & 43,06 & 39,50 & 35,93 & 32,34 & 28,64 & 24,69 & 20,67 & 16,60 & 13,00 & 9,83 & 7,17 \\
\hline 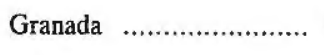 & 40,04 & 45,58 & 53,16 & 49,95 & 45,71 & 42,28 & 38,94 & 35,26 & 31,83 & 28,44 & 24,62 & 20,62 & 16,78 & 12,96 & 9,81 & 6,91 \\
\hline Huelva & 46,78 & 52,48 & 55,72 & 52,25 & 47,93 & 44,12 & 40,60 & 37,08 & 33,51 & 29,63 & 25,83 & 21,77 & 17,87 & 14,16 & 10,73 & 7,58 \\
\hline Jaén & 39,44 & 46,44 & 51,93 & 48,80 & 44,72 & 41,10 & 37,49 & 34,13 & 30,94 & 27,39 & 23,93 & 20,14 & 16,82 & 14,08 & 10,76 & 7,49 \\
\hline 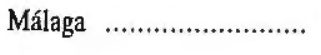 & 40,64 & 46,26 & 52,21 & 48,73 & 44,93 & 41,05 & 37,72 & 34,37 & 30,78 & 27,23 & 23,38 & 20,54 & 17,07 & 13,55 & 10,02 & 6,99 \\
\hline Sevilla & 40,65 & 46,79 & 53,38 & 50,31 & 46,20 & 42,58 & 39,39 & 36,08 & 32,56 & 29,13 & 25,55 & 21,40 & 17,43 & 13,88 & 10,56 & 7,67 \\
\hline TOTAL ANDALUCIA .. & 40,97 & 46,89 & 53,07 & 49,92 & 45,78 & 42,09 & 38,73 & 35,29 & 31,79 & 28,22 & 24,51 & 20,68 & 16,94 & 13,41 & 10,16 & 7,18 \\
\hline ESPAÑA & 43,42 & 48,87 & 53,79 & 50,53 & 46,38 & 42,65 & 39,11 & 35,56 & 31,98 & 28,35 & 24,62 & 20,83 & 17,16 & 13,67 & 10,62 & 7,95 \\
\hline
\end{tabular}


CUADRO III.a (Continuación)

ESPERANZA DE VIDA. MUJERES

ANDALUCIA TOTAL Y PROVINCIAS. ESPAÑA TOTAL

\begin{tabular}{|c|c|c|c|c|c|c|c|c|c|c|c|c|c|c|c|c|}
\hline & 0 & 1 & 5 & 10 & 15 & 20 & 25 & 30 & 35 & 40 & 45 & 50 & 55 & 60 & 65 & 70 \\
\hline \multicolumn{17}{|c|}{$1940-41$} \\
\hline Almería & 51,40 & 57,07 & 57,64 & 53,63 & 49,35 & 45,30 & 41,32 & 37,35 & 33,33 & 29,29 & 25,27 & 21,38 & 17,88 & 14,11 & 10,88 & 7,54 \\
\hline 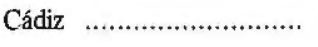 & 50,38 & 56,37 & 56,27 & 52,13 & 47,76 & 43,74 & 39,91 & 36,05 & 32,03 & 28,21 & 24,24 & 20,40 & 16,63 & 13,15 & 10,02 & 7,17 \\
\hline Córdoba & 67,50 & 71,73 & 70,99 & 66,66 & 62,04 & 57,58 & 53,22 & 48,90 & 44,46 & 40,09 & 35,68 & 31,30 & 27,09 & 22,97 & 19,19 & 15,72 \\
\hline Granada & 65,90 & 69,66 & 68,63 & 64,21 & 59,56 & 55,04 & 50,58 & 46,19 & 41,77 & 37,47 & 33,14 & 28,80 & 24,58 & 20,53 & 16,89 & 13,27 \\
\hline Huelva & 71,12 & 74,28 & 72,39 & 67,89 & 63,33 & 58,80 & 54,47 & 50,03 & 45,72 & 41,33 & 36,79 & 32,33 & 28,19 & 23,90 & 19,89 & 16,27 \\
\hline Jaén & 62,56 & 67,50 & 67,41 & 63,19 & 58,53 & 54,06 & 49,77 & 45,58 & 41,27 & 36,90 & 32,62 & 28,37 & 24,21 & 20,17 & 16,49 & 13,10 \\
\hline 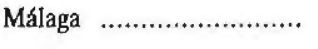 & 65,59 & 72,63 & 70,92 & 66,43 & 61,78 & 57,23 & 52,80 & 48,50 & 44,11 & 39,72 & 35,33 & 30,90 & 26,62 & 22,40 & 18,60 & 15,08 \\
\hline Sevilla & 68,88 & 72,88 & 71,35 & 66,96 & 62,27 & 57,84 & 53,40 & 48,94 & 44,61 & 40,20 & 35,78 & 31,38 & 27,20 & 23,08 & 19,32 & 15,79 \\
\hline TOTAL ANDALUCIA .. & 62,67 & 67,07 & 66,24 & 61,92 & 57,32 & 52,92 & 48,63 & 44,36 & 40,06 & 35,78 & 31,47 & 27,21 & 23,12 & 19,09 & 15,46 & 12,03 \\
\hline ESPAÑA & 53,62 & 58,93 & 58,58 & 54,53 & 50,13 & 45,96 & 41,93 & 37,88 & 33,81 & 29,72 & 25,63 & 21,60 & 17,68 & 13,93 & 10,42 & 7,11 \\
\hline
\end{tabular}


CUADRO III.a (Conclusión)

ESPERANZA DE VIDA. MUJERES

ANDALUCIA TOTAL Y PROVINCIAS. ESPAÑA TOTAL

\begin{tabular}{|c|c|c|c|c|c|c|c|c|c|c|c|c|c|c|c|c|}
\hline & 0 & 1 & 5 & 10 & 15 & 20 & 25 & 30 & 35 & 40 & 45 & 50 & 55 & 60 & 65 & 70 \\
\hline \multicolumn{17}{|c|}{$1975-79$} \\
\hline Almería & 76,41 & 76,83 & 73,01 & 68,10 & 63,18 & 58,28 & 53,35 & 48,51 & 43,69 & 38,90 & 34,16 & 29,48 & 24,99 & 20,59 & 16,40 & 12,87 \\
\hline Cádiz …............................ & 76,07 & 76,19 & 72,42 & 67,52 & 62,60 & 57,70 & 52,79 & 47,89 & 43,08 & 38,28 & 33,59 & 29,05 & 24,63 & 20,35 & 16,37 & 12,86 \\
\hline Córdoba & 77,21 & 78,50 & 74,68 & 69,75 & 64,84 & 59,92 & 55,03 & 50,15 & 45,29 & 40,47 & 35,75 & 31,10 & 26,60 & 22,20 & 18,14 & 14,38 \\
\hline Granada & 76,73 & 77,14 & 73,35 & 68,45 & 63,52 & 58,61 & 53,71 & 48,86 & 44,02 & 39,22 & 34,54 & 29,94 & 25,49 & 21,13 & 17,00 & 13,27 \\
\hline Huelva & 76,51 & 76,83 & 73,00 & 68,09 & 63,18 & 58,27 & 53,36 & 48,49 & 43,64 & 38,90 & 34,17 & 29,66 & 25,15 & 20,79 & 16,61 & 12,95 \\
\hline Jaén & 77,73 & 78,04 & 74,19 & 69,28 & 64,36 & 59,44 & 54,53 & 49,66 & 44,81 & 40,01 & 35,28 & 30,69 & 26,16 & 21,76 & 17,60 & 13,86 \\
\hline Málaga ….......................... & 76,81 & 77,00 & 73,19 & 68,29 & 63,36 & 58,46 & 53,57 & 48,72 & 43,90 & 39,07 & 34,38 & 29,82 & 25,32 & 21,06 & 17,05 & 13,31 \\
\hline Sevilla & 77,48 & 77,48 & 73,66 & 68,75 & 63,83 & 58,92 & 54,03 & 49,14 & 44,33 & 39,50 & 34,83 & 30,26 & 25,78 & 21,49 & 17,47 & 13,69 \\
\hline TOTAL ANDALUCIA ... & 77,01 & 77,26 & 73,45 & 68,54 & 63,63 & 58,72 & 53,82 & 48,94 & 44,12 & 39,31 & 34,61 & 30,03 & 25,56 & 21,23 & 17,16 & 13,46 \\
\hline ESPAN̈A & 77,98 & 78,09 & 74,31 & 69,42 & 64,51 & 59,61 & 54,73 & 49,87 & 45,03 & 40,22 & 35,52 & 30,92 & 26,46 & 22,13 & 18,04 & 14,31 \\
\hline
\end{tabular}




\section{CUADRO III.b}

ESPERANZA DE VIDA. VARONES

ANDALUCIA TOTAL Y PROVINCIAS. ESPAÑA TOTAL

\begin{tabular}{|c|c|c|c|c|c|c|c|c|c|c|c|c|c|c|c|c|}
\hline & 0 & 1 & 5 & 10 & 15 & 20 & 25 & 30 & 35 & 40 & 45 & 50 & 55 & 60 & 65 & 70 \\
\hline \multicolumn{17}{|c|}{$1910-11$} \\
\hline Almería & 37,92 & 43,23 & 50,25 & 47,28 & 42,93 & 39,39 & 35,90 & 32,23 & 28,78 & 25,32 & 21,75 & 18,50 & 15,11 & 11,98 & 9,01 & 6,38 \\
\hline 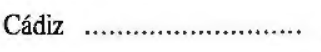 & 38,32 & 44,53 & 49,95 & 46,85 & 42,56 & 38,85 & 35,04 & 31,29 & 27,65 & 24,09 & 20,65 & 17,32 & 13,98 & 11,16 & 8,57 & 6,49 \\
\hline Córdoba & 40,55 & 46,61 & 53,60 & 50,40 & 45,98 & 42,20 & 38,50 & 34,56 & 30,67 & 26,83 & 23,03 & 19,19 & 15,46 & 12,06 & 9,17 & 7,09 \\
\hline Granada & 39,60 & 45,63 & 52,61 & 49,36 & 45,23 & 41,20 & 37,50 & 33,67 & 29,79 & 26,06 & 22,73 & 18,98 & 15,39 & 12,21 & 9,47 & 6,84 \\
\hline Huelva & 43,69 & 49,73 & 52,84 & 49,08 & 44,79 & 40,70 & 37,07 & 33,47 & 29,67 & 26,03 & 22,46 & 18,68 & 14,96 & 11,98 & 9,16 & 6,54 \\
\hline Jaén & 39,11 & 45,75 & 52,15 & 49,01 & 44,91 & 41,07 & 37,43 & 33,83 & 30,16 & 26,60 & 23,02 & 19,06 & 15,39 & 12,00 & 8,96 & 6,09 \\
\hline 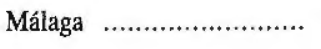 & 39,31 & 44,92 & 50,99 & 47,48 & 43,21 & 39,28 & 35,78 & 31,95 & 28,10 & 24,33 & 20,50 & 18,51 & 15,29 & 12,24 & 9,07 & 6,63 \\
\hline 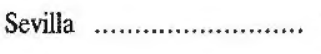 & 38,47 & 45,08 & 51,55 & 48,37 & 44,14 & 40,33 & 37,00 & 33,38 & 29,67 & 26,16 & 22,84 & 18,78 & 15,24 & 12,25 & 9,47 & 6,94 \\
\hline TOTAL ANDALUCIA .. & 39,44 & 45,55 & 51,80 & 48,56 & 44,30 & 40,46 & 36,86 & 33,12 & 29,38 & 25,75 & 22,19 & 18,68 & 15,15 & 12,03 & 9,16 & 6,69 \\
\hline ESPAÑA & 41,92 & 47,89 & 53,10 & 49,80 & 45,56 & 41,78 & 38,26 & 34,57 & 30,82 & 27,14 & 23,49 & 19,99 & 16,59 & 13,50 & 10,79 & 8,66 \\
\hline
\end{tabular}


CUADRO III.b (Continuación)

ESPERANZA DE VIDA. VARONES

ANDALUCIA TOTAL Y PROVINCIAS. ESPAÑA TOTAL

\begin{tabular}{|c|c|c|c|c|c|c|c|c|c|c|c|c|c|c|c|c|}
\hline & 0 & 1 & 5 & 10 & 15 & 20 & 25 & 30 & 35 & 40 & 45 & 50 & 55 & 60 & 65 & 70 \\
\hline \multicolumn{17}{|c|}{$1940-41$} \\
\hline Almería & 43,82 & 49,38 & 49,41 & 45,39 & 40,88 & 36,78 & 33,42 & 30,27 & 26,71 & 23,23 & 19,96 & 16,75 & 13,64 & 10,97 & 8,26 & 5,87 \\
\hline Cádiz …........................... & 41,83 & 47,47 & 46,67 & 42,55 & 38,11 & 34,03 & 30,68 & 27,36 & 23,63 & 20,11 & 16,81 & 14,06 & 11,37 & 9,06 & 7,22 & 5,41 \\
\hline Córdoba ........................... & 58,99 & 62,99 & 61,58 & 57,18 & 52,58 & 48,12 & 44,16 & 40,67 & 36,83 & 32,96 & 29,05 & 25,33 & 21,76 & 18,17 & 15,02 & 12,23 \\
\hline Granada ........................... & 60,38 & 64,30 & 63,04 & 58,57 & 53,97 & 49,52 & 45,40 & 41,39 & 37,30 & 33,17 & 29,12 & 25,26 & 21,37 & 17,80 & 14,65 & 11,97 \\
\hline Huelva & 63,65 & 66,41 & 64,06 & 59,61 & 54,96 & 50,43 & 46,31 & 42,34 & 38,20 & 34,10 & 30,21 & 26,56 & 23,05 & 19,83 & 16,95 & 14,30 \\
\hline Jaén & 53,63 & 58,49 & 57,97 & 53,69 & 49,06 & 44,70 & 41,00 & 37,50 & 33,90 & 30,24 & 26,58 & 22,96 & 19,43 & 16,12 & 13,06 & 10,22 \\
\hline Málaga .............................. & 62,70 & 66,03 & 63,89 & 59,48 & 54,80 & 50,28 & 46,22 & 42,27 & 38,41 & 34,44 & 30,40 & 26,63 & 22,85 & 19,18 & 15,84 & 12,62 \\
\hline Sevilla & 61,63 & 65,42 & 63,72 & 59,31 & 54,71 & 50,32 & 46,03 & 41,84 & 37,57 & 33,39 & 29,47 & 25,73 & 22,07 & 18,63 & 15,34 & 12,27 \\
\hline TOTAL ANDALUCLA ... & 54,90 & 59,21 & 57,98 & 53,64 & 49,05 & 44,68 & 40,82 & 37,10 & 33,19 & 29,31 & 25,54 & 22,01 & 18,53 & 15,29 & 12,34 & 9,65 \\
\hline ESPAN̈A & 45,51 & 49,83 & 49,07 & 45,01 & 40,61 & 36,65 & 33,52 & 30,44 & 27,19 & 23,87 & 20,52 & 17,40 & 14,36 & 11,67 & 9,12 & 6,99 \\
\hline
\end{tabular}


CUADRO III.b (Conclusión)

ESPERANZA DE VIDA. VARONES

ANDALUCIA TOTAL Y PROVINCIAS. ESPAÑA TOTAL

\begin{tabular}{|c|c|c|c|c|c|c|c|c|c|c|c|c|c|c|c|c|}
\hline & 0 & 1 & 5 & 10 & 15 & 20 & 25 & 30 & 35 & 40 & 45 & 50 & 55 & 60 & 65 & 70 \\
\hline \multicolumn{17}{|c|}{ 1975-79 } \\
\hline Almería & 70,73 & 71,20 & 67,44 & 62,58 & 57,71 & 52,99 & 48,29 & 43,64 & 38,92 & 34,19 & 29,73 & 25,34 & 21,24 & 17,37 & 13,82 & 10,80 \\
\hline 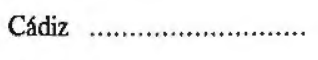 & 68,85 & 69,31 & 65,59 & 60,72 & 55,82 & 50,99 & 46,25 & 41,49 & 36,80 & 32,14 & 27,70 & 23,42 & 19,49 & 15,73 & 12,47 & 9,86 \\
\hline Córdoba & 71,62 & 72,31 & 68,48 & 63,58 & 58,69 & 53,88 & 49,08 & 44,29 & 39,62 & 34,90 & 30,34 & 25,96 & 21,81 & 17,81 & 14,23 & 11,23 \\
\hline Granada ......................... & 71,46 & 72,03 & 68,26 & 63,38 & 58,48 & 53,68 & 48,91 & 44,15 & 39,43 & 34,76 & 30,26 & 25,79 & 21,65 & 17,65 & 14,24 & 11,31 \\
\hline (n).................... & 69,38 & 69,93 & 66,15 & 61,28 & 56,38 & 51,57 & 46,83 & 42,08 & 37,39 & 32,68 & 28,19 & 23,90 & 19,81 & 15,98 & 12,39 & 9,61 \\
\hline Jaén & 72,12 & 72,79 & 68,98 & 64,11 & 59,24 & 54,42 & 49,64 & 44,88 & 40,16 & 35,44 & 30,86 & 26,48 & 22,30 & 18,33 & 14,67 & 11,68 \\
\hline Málaga & 70,17 & 70,62 & 66,82 & 61,92 & 57,03 & 52,25 & 47,52 & 42,80 & 38,08 & 33,41 & 28,95 & 24,70 & 20,78 & 17,08 & 13,79 & 11,00 \\
\hline Sevilla $\ldots \ldots \ldots \ldots \ldots \ldots \ldots \ldots$ & 70,57 & 70,76 & 67,01 & 62,13 & 57,23 & 52,42 & 47,64 & 42,84 & 38,09 & 33,38 & 28,90 & 24,66 & 20,72 & 17,00 & 13,59 & 10,74 \\
\hline TOTAL ANDALUCIA .. & 70,62 & 71,07 & 67,29 & 62,41 & 57,52 & 52,72 & 47,96 & 43,20 & 38,48 & 33,79 & 29,30 & 24,98 & 20,95 & 17,12 & 13,69 & 10,84 \\
\hline ESPAÑA & 71,60 & 71,89 & 68,14 & 63,28 & 58,40 & 53,64 & 48,93 & 44,21 & 39,50 & 34,81 & 30,33 & 26,00 & 21,95 & 18,09 & 14,58 & 11,60 \\
\hline
\end{tabular}




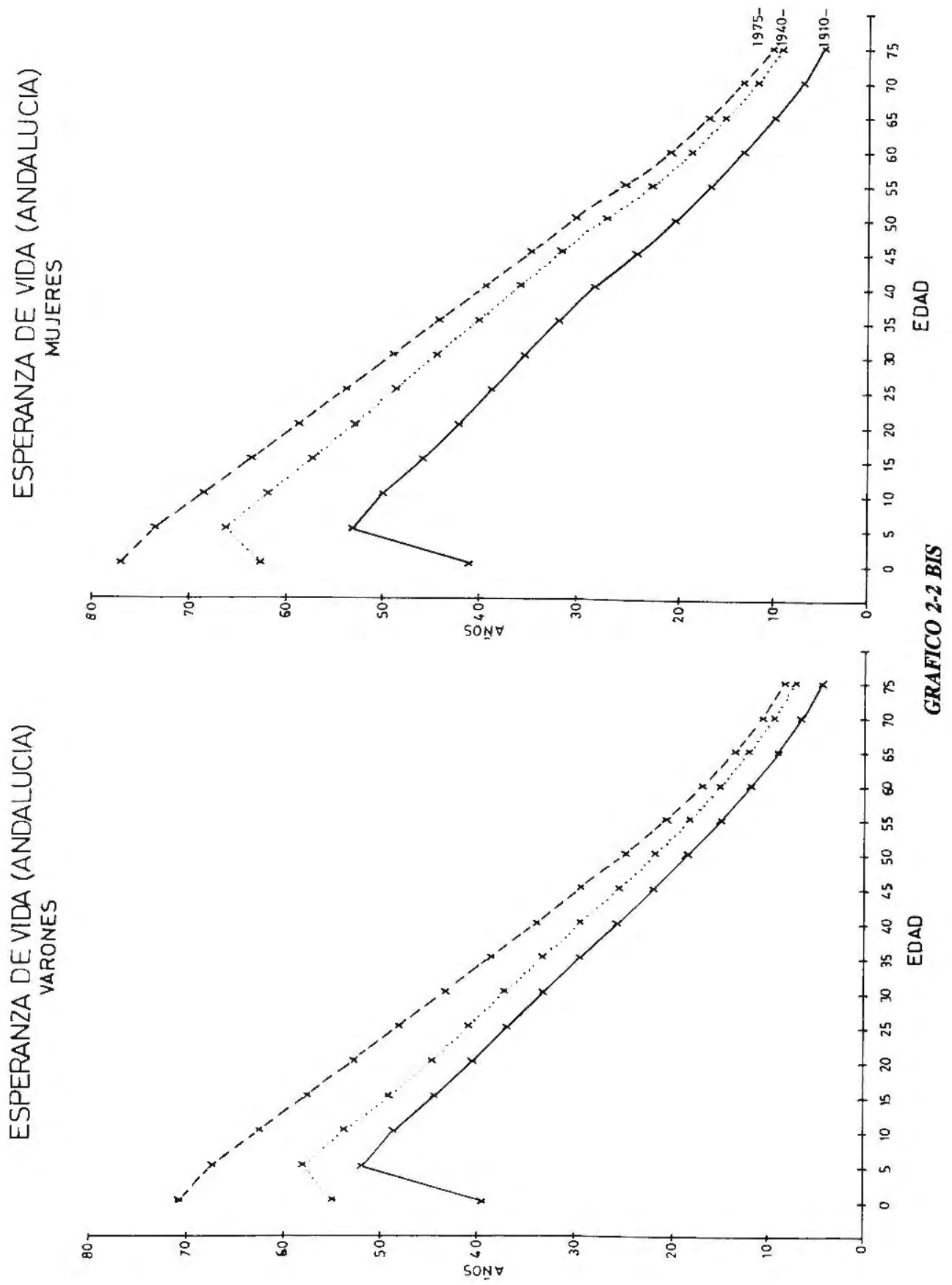


universal, de una mayor fortaleza de la mujer frente a la vida. Por lo demás, la curva que expresa la esperanza de vida para el sexo femenino obtenía un perfil muy semejante en el caso de los grupos femeninos para Andalucía y España, siendo la española levemente más alta que la correspondiente a Andalucía en los primeros grupos de edades.

En definitiva, y ello es lo que nos interesa, la curva de esperanza de vida para 1910-11 en Andalucía (también España) presentaba un modelo teórico caracterizado por unas escasas expectativas en los primeros años de vida para, alcanzar los máximos valores en los grupos jóvenes e ir progresivamente disminuyendo hasta las etapas finales de la vida. Con arreglo al comportamiento de la gráfica pues, podemos afirmar que efectivamente nos encontramos ante un conjunto demográfico que manifestaba un horizonte de vida propio de las poblaciones con régimen demográfico antiguo.

Para 1941 la observación de las gráficas correspondientes nos llevan a subrayar los siguientes hechos:

En primer lugar sobresale el tremendo ascenco experimentado por la esperanza de vida en todas las edades para la población andaluza, ascenso, sin duda, el mayor de todo el siglo para el grupo femenino, lo que nos anima a argumentar, nuevamente, la mayor resistencia biológica de la mujer, hecho que posibilitó, probablemente, un éxito mucho más rápido en la reducción de la mortalidad exógena.

Este aumento de la espernaza de vida resulta especialmente llamativa tanto en el caso de la esperanza de vida al nacer como, sobre todo en la edad de 70 años, momento en el que la esperanza de vida experimentó prácticamente una duplicación con respecto a la correspondiente a 1910-11, esto es, se duplicaron los años potenciales o posible a vivir.

En tercer lugar, conviene llamar la atención en un hecho ya apuntado, esto es, que el aumento en la esperanza de vida pudo detectarse en el conjunto demográfico andaluz, para ambos sexos - aunque mucho más elevado para el caso femenino, como acabamos de ver-. Sin embargo, esta tendencia a la recuperación no fue compartida por el conjunto masculino a escala nacional, conjunto que manifestó un horizonte de expectativa de vida inferior incluso al registrado para 1910-11. Tan sólo pudo observarse una recuperación para dicho conjunto en el cálculo correspondiente a la esperanza de vida al nacer, fenómeno que se explica, como ya antes se señaló, por un descenso acusado de la mortalidad infantil en dicho conjunto demográfico. Esta circunstancia nos remite nuevamente a la idea, ya señalada, a saber, que la población andaluza, dado probablemente su carácter marcadamente rural, pudo defenderse de forma más eficaz, a nivel coyuntural, de los tremendos rigores de la postguerra. 
En definitiva, las curvas que representan los horizontes de vida para 1940-41 presentaban, según nuestra opinión, un perfil algo más evolucionado, lo que nos indica unos niveles en las expectativas de vida propios de los conjuntos demográficos en vias de transición entre un régimen demográfico antiguo, y otro marcadamente evolucionado.

Las últimas series, correspondientes a 1975-79, viene a indicarnos que, efectivamente, la esperanza de vida seguía aumentado para todas las edades, siendo especialmente significativa la recuperación experimentada en la esperanza de vida al nacer y la correspondiente al grupo 1-4 años, habiendo alcanzado los 72 años para el grupo masculino, y los 78 en el femenino, parámetros semejantes a los registrados en los países desarrollados. Puede afirmarse, que el alto riesgo que conllevaba este grupo de edades había prácticamente desaparecido, gracias a los avances médicos y condiciones de vida en general. Sigue manteniéndose, como tónica general, y como reiteradamente venimos poniendo de manifiesto, una mayor esperanza de vida para el grupo femenino.

De igual forma habría que señalar, que la recuperación fue durante este período de tiempo, mayor para el grupo masculino, localizándose en estos años la mayor elevación de la esperanza de vida en lo que va de siglo. Los argumentos que explican este comportamiento no podían ser otros: la eliminación de los riesgos de mortalidad masculina imputables a los comportamientos post-bélicos de una parte, asi como un éxito más lento en la lucha contra la mortalidad exógena para los varones de otra, son las tesis que explican los comportamientos ya señalados.

Por último y antes de finalizar con nuestros comentarios sobre la esperanza de vida para este período, no podemos dejar de citar dos nuevos hechos. El primero de ellos consiste en destacar la escasa elevación experimentada en la esperanza de vida a la edad 70-75 años para ambos conjuntos y sexos. Ello nos induce a pensar que la elevación del horizonte de vida a estas edades ha tocado techo, tendiendo a estancarse o, a lo sumo, oscilar ligeramente. En este sentido y antes de pronunciarse, habrá que estar atentos a futuras evoluciones.

La segunda idea consiste, en la comprobación de que las esperanzas de vida a distintas edades para Andalucía, eran prácticamente las mismas que las obtenidas para el resto del país, lo que señalan a ambos conjuntos demográficos como agregados definidos con arreglo a las mismas leyes de mortalildad.

En definitiva, la observación llevada a cabo acerca de la esperanza de vida en las poblaciones andaluza y española para 1979, nos ha dibujado unas gráficas de horizontes de vida que reflejan el perfíl propio y característico de las poblaciones con régimen demográfico evolucionado, modelo que se resuelve en el trazo de una línea recta, sin las inflexiones correspondientes a las primeras edades, cuya pendiente viene a expresarnos la disminución progresiva de 
las esperanzas de vida de acuerdo con las leyes biológicas, esto es, muy altas en el momento de nacer con una disminución progresiva de las expectativas conforme se avanza a través de los distintos grupos de edades.

\section{LAS PROBABILIDADES DE DEFUNCION (Qx)}

Las probabilidades de muerte en una tabla de mortalidad se expresan mediante los denominados cocientes de mortalidad (Q), cálculos a través de los cuales se obtiene la posibilidad de que los efectivos de un grupo de edad determinada fallezcan antes de alcanzar su siguiente aniversario.

Según el modelo teórico tradicional, la curva que indica las posibilidades de defunción para cada grupo de edad se resuelve en una alta probabilidad de defunción en las primeras edades, para decrecer rápidamente hasta lograr el mínimo en torno a los quince años. A partir de esos grupos de edades las probabilidades de defunción van paulatinamente incrementándose hasta alcanzar los máximos valores en los grupos terminales de cualquier conjunto demográfico.

Si observamos la trayectoria de las líneas de probabilidades de defunción para la población andaluza (vid. gráfico n. ${ }^{\circ}$ 3) y si a su vez tenemos presente los datos correspondientes (vid. tablas de mortalidad, columnas correspondientes a Q), podemos destacar los siguientes hechos:

En 1910-11, las líneas correspondientes a los cocientes de mortalidad, tanto masculinos como femeninos, se adecuaban perfectamente al modelo teórico descrito, de tal modo que la posibilidad de alcanzar aniversarios sucesivos en los 5 primeros años de la vida eran relativamente escasas. Seguidamente, el riesgo de defunción disminuía situándose en el mínimo en tomo a los 15-19 años para, a partir de dicho grupo de edad ir aumentando paulatinamente hasta alcanzar los valores más altos en torno a los 65 años. Conviene subrayar, no obstante, la comprobación de que las probabilidades de defunción eran relativamente más bajas - comparativamente hablando- a partir de los 40 años para el grupo femenino. Junto a ello destaca, refiriéndonos ahora a todo el conjunto, la existencia de fuertes riesgos de mortalidad en todos los grupos de edades, incluso para aquellos años en los que los riesgos de mortalidad son mínimos. La existencia de estas altas posibilidades de defunción en todas las edades, manifestaba la existencia de sucesos fatales de naturaleza exógena, imputables directamente a la existencia de unas condiciones de salubridad, sanidad en general y nivel de vida, altamente deficientes.

Las líneas correspondientes a 1941 presentaban, como tónica general, un nivel considerablemente mas bajo que las correspondientes al período anterior, hecho que viene a indicar, obviamente, un descenso en las probabilidades 
CUADRO IV

TABLAS DE VIDA. ANDALUCIA (1910-11)

\begin{tabular}{|c|c|c|c|c|c|c|c|c|c|}
\hline \multirow[b]{2}{*}{$\begin{array}{c}\text { Edad a } \\
\text { comienzo } \\
\text { de Período } \\
\mathbf{x} \\
\end{array}$} & \multirow[b]{2}{*}{$\begin{array}{c}\text { Anchura } \\
\text { del } \\
\text { Período } \\
\text { n } \\
\end{array}$} & \multicolumn{4}{|c|}{ VARONES } & \multicolumn{4}{|c|}{ MUJERES } \\
\hline & & $\begin{array}{l}\text { Cocientes } \\
\text { de } \\
\text { Mortalidad } \\
{ }_{n} q_{x} \\
\end{array}$ & $\begin{array}{c}\text { Super- } \\
\text { Vivientes } \\
\text { (a edad } x \text { ) } \\
\mathrm{l}_{\mathrm{z}} \\
\end{array}$ & $\begin{array}{c}\text { Personas } \\
\text {-Año } \\
{ }_{n} T_{x}\end{array}$ & $\begin{array}{c}\text { Esperanza } \\
\text { de Vida } \\
\text { (a edad } x \text { ) } \\
e_{x} \\
\end{array}$ & $\begin{array}{l}\text { Cocientes } \\
\text { de } \\
\text { Mortalidad } \\
{ }_{n} q_{x} \\
\end{array}$ & $\begin{array}{c}\text { Super- } \\
\text { Vivientes } \\
(\text { a edad } x) \\
l_{x} \\
\end{array}$ & $\begin{array}{c}\text { Personas } \\
\text {-Año } \\
{ }_{n} T_{x}\end{array}$ & $\begin{array}{c}\text { Esperanza } \\
\text { de Vida } \\
\text { (a edad } x \text { ) } \\
\mathrm{e}_{x} \\
\end{array}$ \\
\hline 0 & 1 & 0.15307 & 100000 & 86224 & 39,44 & 0.14484 & 100000 & 86964 & 40,97 \\
\hline 1 & 4 & 0.18895 & 84693 & 299725 & 45,55 & 0.18335 & 85516 & 303806 & 46,89 \\
\hline 5 & 5 & 0.03425 & 68690 & 337099 & 51,80 & 0.03517 & 69837 & 342552 & 53,07 \\
\hline 10 & 5 & 0.01593 & 66338 & 329258 & 48,56 & 0.01790 & 67380 & 334128 & 49,92 \\
\hline 15 & 5 & 0.02723 & 65281 & 322583 & 44,30 & 0.02972 & 66174 & 326644 & 45,78 \\
\hline 20 & 5 & 0.03550 & 63503 & 311768 & 40,46 & 0.03958 & 64208 & 314558 & 42,09 \\
\hline 25 & 5 & 0.03551 & 61249 & 300807 & 36,86 & 0.04133 & 61666 & 301960 & 38,72 \\
\hline 30 & 5 & 0.03958 & 59074 & 289758 & 33,12 & 0.04386 & 59118 & 289365 & 35,29 \\
\hline 35 & 5 & 0.04883 & 56736 & 277307 & 29,38 & 0.04701 & 56525 & 276512 & 31,79 \\
\hline 40 & 5 & 0.05896 & 53965 & 262509 & 25,75 & 0.04811 & 53868 & 263377 & 28,22 \\
\hline 45 & 5 & 0.07058 & 50784 & 245674 & 22,19 & 0.05093 & 51276 & 250373 & 24,51 \\
\hline 50 & 5 & 0.08446 & 47199 & 226628 & 18,68 & 0.06495 & 48664 & 235895 & 20,68 \\
\hline 55 & 5 & 0.13000 & 43213 & 202582 & 15,15 & 0.09337 & 45504 & 217322 & 16,94 \\
\hline 60 & 5 & 0,18448 & 37595 & 171330 & 12,03 & 0.13888 & 41255 & 192524 & 13,41 \\
\hline 65 & 5 & 0.27777 & 30660 & 132859 & 9,16 & 0.21162 & 35526 & 159585 & 10,16 \\
\hline 70 & 5 & 0.41024 & 22143 & 88460 & 6,69 & 0.37302 & 28008 & 114442 & 7,18 \\
\hline 75 & $\ldots$ & 1.00000 & 13059 & 59588 & 4,56 & 1.00000 & 17560 & 86764 & 4,94 \\
\hline
\end{tabular}


CUADRO V

TABLAS DE VIDA. ANDALUCIA (1940-41)

\begin{tabular}{|c|c|c|c|c|c|c|c|c|c|}
\hline \multirow[b]{2}{*}{$\begin{array}{c}\text { Edad a } \\
\text { comienzo } \\
\text { de Período } \\
\mathbf{x}\end{array}$} & \multirow[b]{2}{*}{$\begin{array}{c}\text { Anchura } \\
\text { del } \\
\text { Período } \\
\mathbf{n}\end{array}$} & \multicolumn{4}{|c|}{ VARONES } & \multicolumn{4}{|c|}{ MUJERES } \\
\hline & & $\begin{array}{l}\text { Cocientes } \\
\text { de } \\
\text { Mortalidad } \\
{ }_{n} 9 x \\
\end{array}$ & $\begin{array}{c}\text { Super- } \\
\text { Vivientes } \\
\text { (a edad } x \text { ) } \\
1_{x}\end{array}$ & $\begin{array}{l}\text { Personas } \\
\text {-Año } \\
{ }_{n} T_{x}\end{array}$ & $\begin{array}{c}\text { Esperanza } \\
\text { de Vida } \\
\text { (a edad } x \text { ) } \\
e_{x}\end{array}$ & $\begin{array}{c}\text { Cocientes } \\
\text { de } \\
\text { Mortalidad } \\
{ }_{n} q_{x} \\
\end{array}$ & $\begin{array}{c}\text { Super- } \\
\text { Vivientes } \\
\text { (a edad } x \text { ) } \\
1_{x} \\
\end{array}$ & $\begin{array}{c}\text { Personas } \\
\text {-Año } \\
{ }_{n} \mathrm{~T}_{x}\end{array}$ & $\begin{array}{c}\text { Esperanza } \\
\text { de Vida } \\
\text { (a edad } x) \\
e_{x}\end{array}$ \\
\hline 0 & 1 & 0.0884 & 100000 & 92040 & 54,90 & 0.07939 & 100000 & 92855 & 62,67 \\
\hline 1 & 4 & 0.04573 & 91156 & 354453 & 59,21 & 0.04618 & 92061 & 357871 & 67,07 \\
\hline 5 & 5 & 0.01177 & 86987 & 432173 & 57,98 & 0.01049 & 87810 & 436561 & 66,24 \\
\hline 10 & 5 & 0.00792 & 85964 & 428252 & 53,64 & 0.00683 & 86889 & 433078 & 61,92 \\
\hline 15 & 5 & 0.01352 & 85283 & 423935 & 49,05 & 0.01090 & 86295 & 429453 & 57,32 \\
\hline 20 & 5 & 0.02639 & 84130 & 414987 & 44,68 & 0.01375 & 85354 & 423779 & 52,92 \\
\hline 25 & 5 & 0.03227 & 81910 & 402940 & 40,82 & 0.01563 & 84181 & 417615 & 48,63 \\
\hline 30 & 5 & 0.03064 & 79266 & 390503 & 37,10 & 0.01637 & 82865 & 411070 & 44,36 \\
\hline 35 & 5 & 0.03541 & 76838 & 377930 & 33,19 & 0.01898 & 81509 & 403985 & 40,06 \\
\hline 40 & 5 & 0.04428 & 74117 & 363036 & 29,31 & 0.02050 & 79962 & 396038 & 35,78 \\
\hline 45 & 5 & 0.06019 & 70835 & 344368 & 25,54 & 0.02511 & 78322 & 387088 & 31,47 \\
\hline 50 & 5 & 0.07277 & 66571 & 321473 & 22,01 & 0.03550 & 76356 & 375408 & 27,21 \\
\hline 55 & 5 & 0.09946 & 61727 & 293900 & 18,53 & 0.04529 & 73645 & 360220 & 23,12 \\
\hline 60 & 5 & 0.13910 & 55588 & 259381 & 15,29 & 0.07649 & 70310 & 338641 & 19,09 \\
\hline 65 & 5 & 0.19217 & 47855 & 217206 & 12,34 & 0.10921 & 64932 & 307639 & 15,46 \\
\hline 70 & 5 & 0.27752 & 38659 & 167010 & 9,65 & 0.20148 & 57840 & 260651 & 12,03 \\
\hline 75 & $\ldots$ & 1.00000 & 27930 & 206113 & 7,38 & 1.00000 & 46187 & 435232 & 9,42 \\
\hline
\end{tabular}


CUADRO VI

TABLAS DE VIDA. ANDALUCIA (1975-79)

\begin{tabular}{|c|c|c|c|c|c|c|c|c|c|}
\hline \multirow[b]{2}{*}{$\begin{array}{c}\text { Edad a } \\
\text { comienzo } \\
\text { de Período } \\
x \\
\end{array}$} & \multirow[b]{2}{*}{$\begin{array}{c}\text { Anchura } \\
\text { del } \\
\text { Período } \\
\text { n }\end{array}$} & \multicolumn{4}{|c|}{ VARONES } & \multicolumn{4}{|c|}{ MUJERES } \\
\hline & & $\begin{array}{c}\text { Cocientes } \\
\text { de } \\
\text { Mortalidad } \\
{ }_{n} q_{x} \\
\end{array}$ & $\begin{array}{c}\text { Super- } \\
\text { Vivientes } \\
\text { (a edad } x \text { ) } \\
l_{x}\end{array}$ & $\begin{array}{c}\text { Personas } \\
\text {-An̈o } \\
{ }_{\mathrm{n}} \mathrm{T}_{x} \\
\end{array}$ & $\begin{array}{c}\text { Esperanza } \\
\text { de Vida } \\
\text { (a edad } \mathrm{x} \text { ) } \\
\mathrm{e}_{\mathrm{x}} \\
\end{array}$ & $\begin{array}{c}\text { Cocientes } \\
\text { de } \\
\text { Mortalidad } \\
{ }_{n} q_{x} \\
\end{array}$ & $\begin{array}{c}\text { Super- } \\
\text { Vivientes } \\
\text { (a edad } \mathrm{x}) \\
\mathrm{l}_{\mathrm{x}}\end{array}$ & $\begin{array}{c}\text { Personas } \\
\text {-Año } \\
{ }_{n} T_{x}\end{array}$ & $\begin{array}{c}\text { Esperanza } \\
\text { de Vida } \\
\text { (a edad } x \text { ) } \\
e_{x}\end{array}$ \\
\hline 0 & 1 & 0.02017 & 100000 & 98185 & 70,62 & 0.01604 & 100000 & 98556 & 77,01 \\
\hline 1 & 4 & 0.00323 & 97983 & 391160 & 71,07 & 0.00252 & 98396 & 392979 & 77,26 \\
\hline 5 & 5 & 0.00185 & 97667 & 487845 & 67,29 & 0.00130 & 98148 & 490396 & 73,45 \\
\hline 10 & 5 & 0.00175 & 97486 & 487037 & 62,41 & 0.00125 & 98020 & 489820 & 68,54 \\
\hline 15 & 5 & 0.00364 & 97315 & 485815 & 57,52 & 0.00150 & 97898 & 489174 & 63,63 \\
\hline 20 & 5 & 0.00474 & 96961 & 483633 & 52,72 & 0.00180 & 97751 & 488307 & 58,72 \\
\hline 25 & 5 & 0.00529 & 96501 & 481231 & 47,96 & 0.00245 & 97575 & 487278 & 53,82 \\
\hline 30 & 5 & 0.00693 & 95991 & 478358 & 43,20 & 0.00374 & 97336 & 485807 & 48,94 \\
\hline 35 & 5 & 0.00852 & 95326 & 474761 & 38,48 & 0.00459 & 96972 & 483836 & 44,12 \\
\hline 40 & 5 & 0.01613 & 94514 & 469061 & 33,79 & 0.00812 & 96527 & 480832 & 39,31 \\
\hline 45 & 5 & 0.02496 & 92989 & 459607 & 29,30 & 0.01292 & 95743 & 475871 & 34,61 \\
\hline 50 & 5 & 0.04167 & 90668 & 444461 & 24,98 & 0.01918 & 94506 & 468271 & 30,03 \\
\hline 55 & 5 & 0.05988 & 86890 & 421962 & 20,95 & 0.02831 & 92693 & 457170 & 25,56 \\
\hline 60 & 5 & 0.09742 & 81687 & 389335 & 17,12 & 0.04764 & 90069 & 440049 & 21,23 \\
\hline 65 & 5 & 0.16250 & 73729 & 339891 & 13,69 & 0.08185 & 85778 & 412042 & 17,16 \\
\hline 70 & 5 & 0.24390 & 61748 & 271842 & 10,84 & 0.14725 & 78757 & 365375 & 13,46 \\
\hline 75 & $\cdots$ & 1.00000 & 46688 & 397342 & 8,51 & 1.00000 & 67160 & 694811 & 10,35 \\
\hline
\end{tabular}



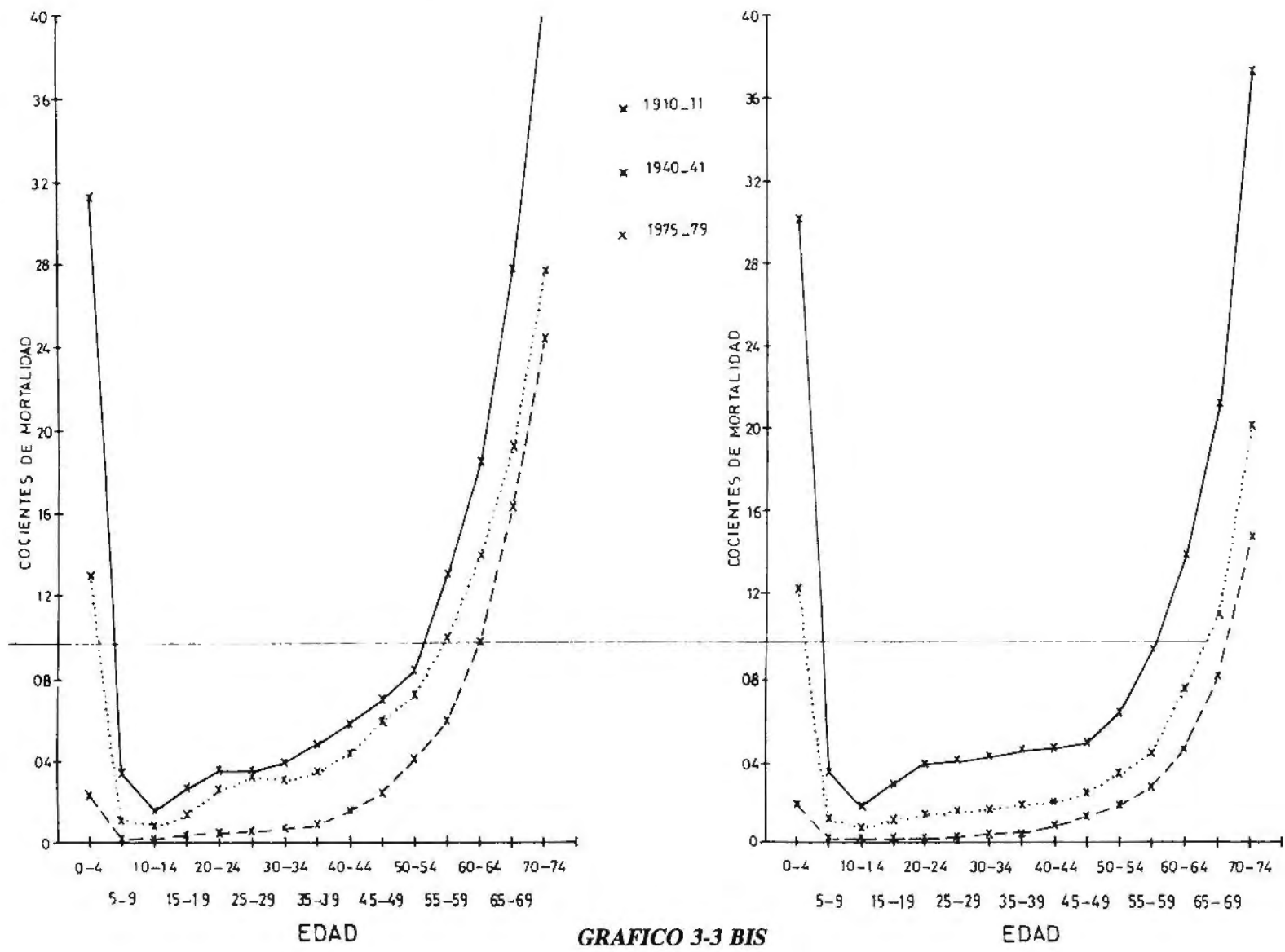

$\times 1910-11$

$\times$ 1940_41

$\times \quad 975 \_79$ 
de defunción para todas las edades; esta disminución se manifestaba de una manera más expresiva, en el grupo femenino, llegando a convertirse en el suceso demográfico —en cuanto a la mortalidad se refiere, naturalmente-, más significativo de todo el período examinado. La curva correspondiente a los efectivos masculinos, por el contrario, mantenía unos niveles de riesgo mucho más altos, en especial en los grupos correspondientes a los 25-35 años, en los que las probabilidades de defunción para esos años eran prácticamente coincidentes con los registrados en 1910-11.

Por último, los cocientes de mortalidad resultantes del examen para 1979 y para ambos sexos, dibujaban unos perfíles ciertamente diferentes a los obtenidos en los cortes temporales anteriores, caracterizándose en estas últimas fechas por una fortísima disminución en las probabilidades de defunción para la población andaluza en todas las edades. En las primeras edades de la vida esta disminución bien ha podido calificarse de espectacular, lo que se explica a través del importantísimo descenso acaecido en la mortalidad infantil. Del mismo modo y también para estas fechas, se registraron cocientes de mortalidad prácticamente nulos hasta los 35 años para, a partir de estos momentos incrementarse rápidamente, aunque dibujándose siempre las líneas por debajo de las calculadas para años anteriores. El descenso en las probabilidades de defunción ha sido en este último período, mayor para el caso masculino.

\section{CONCLUSIONES}

A lo largo de las páginas anteriores hemos expuesto, de forma elemental y sintética, la utilidad de las tablas de mortalidad a la hora de describir los acontecimientos que definen las leyes de mortalidad de un conjunto demográfico cualquiera, en este caso las correspondientes a la población andaluza.

Las tablas de mortalidad se completan mediante el cálculo denominada «columna $S x »$, que expresa, en última instancia, el número de supervivientes alcanzado en cada edad o grupo de edades y para cada período estudiado. Sin embargo, hemos prescindido conscientemente de su comentario (no de su cálculo, que puede consultarse en las tablas de mortalidad) por entender que su descripción tan sólo vendría a confirmar todo lo descrito, no aportando ningún nuevo elemento de juicio a nuestro análisis.

En definitiva, las tablas de mortalidad elaboradas para la población andaluza en los momentos correspondientes a 1910-11, 1940-41, y 1975-79, han venido a indicarnos cómo y de qué manera se han modificado las leyes de mortalidad correspondientes a nuestra comunidad autónoma, pudiendo afirmar, en este sentido, que la transformación radical de las pautas de mortalidad se han ido efectuando de forma lineal y progresiva a lo largo de todo el siglo. Los 
indicadores correspondientes al período inmediatamente posterior a la guerra civil verdaderamente han supuesto una alteración importante al modelo evolutivo, aunque siempre la modificación ha sido mucho más suave que la correspondiente al conjunto nacional.

Puede afirmarse, pues, que la paulatina modificación en los modos de vida de la población andaluza ha sido la causa fundamental a la que ha de imputársele la transformación de unas leyes de mortalidad propias de poblaciones con régimen demográfico antiguo, por otras correspondiente a los modelos que definen a las poblaciones evolucionadas. Ello no significa, naturalmente, que los niveles de renta y calidad de vida de los andaluces sean los propios de los países desarrollados. Baste recordar, en este sentido, las modificaciones tajantes en los modelos de mortalidad obtenidos para los países subdesarrolaldos. Tan sólo hemos querido subrayar, por ello, que la lucha contra la mortalidad llevada a cabo a todo lo largo del siglo en la población andaluza, ha arrojado unos resultados ciertamente satisfactorios y que iguala definitivamente sus leyes de mortalidad a las correspondinetes al conjunto nacional. Sin embargo, el modelo obtenido resulta, cuando menos, peculiar, no ajustándose de forma rigurosa al denominado «de transición demográfica». Por contra, la evolución de la mortalidad señala precisamente como una excepción a dicho modelo.

\section{APENDICE}

Breve descripción de la Metodología y nomenclatura empleadas en el cálculo de las funciones biométricas de las Tablas de Vida a partir de las Tasas de mortalidad específicas por edad (agrupamiento quinquenal). Nomenclatura:

Los subíndices representan:

x La edad de entrada en el grupo de edad.

n La longitud temporal del intérvalo o anchura del grupo de edad.

Por ejemplo un parámetro cualquier «P» que estuviera subindicado con $(\mathrm{n}, \mathrm{x})$, de esta manera " $\mathrm{P}_{\mathrm{x}}$ " haría referencia a una medida cualquiera que afectaría al grupo de edad $[x, x+n)$ integrado por personas de $x$ a $x+n-1$ años cumplidos, si $x=10$ y $n=5$ se refeire sobre el grupo de edad de 10-14 años cumplidos.

Las funciones de las Tablas de vida las representaremos:

${ }_{n} \mathbf{M}_{\mathbf{x}}$ Tasa promedio de Mortalidad Interanual para edad $[\mathrm{x}, \mathrm{x}+\mathrm{n})$.

${ }_{n} m_{x}$ Tasa promedio de mortalidad, pero ahora referida a una generación ya sea o no ficticia. 
La diferencia entre estos dos conceptos se puede comprender mejor sobre el diagrama de Lexis en función del área del diagrama que cada una de las medidas representa:
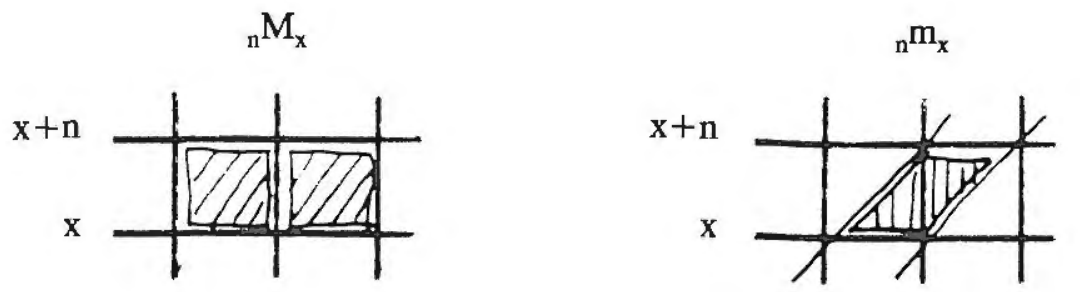

${ }_{n} q_{x}$ Cocientes o Riesgo de fallecer en el rango de edad $[x, x+n)$, habiendo llegado vivo a la edad $\mathrm{x}$.

$\mathrm{l}_{\mathrm{x}} \quad$ Número absoluto de supervivientes a edad $\mathrm{x}$ de una cohorte inicial de tamaño fijado (i.e. $l_{o}=100.000$ ) sobre la que han actuado los distintos riesgos de mortalidad de la Tabla en las edades anteriores a $\mathbf{x}$.

${ }_{n} d_{x} \quad$ Número absoluto de fallecidos de la cohorte inicial (i.e. $1_{0}=100.000$ ) en el rango de edad $[\mathrm{x}, \mathrm{x}+\mathrm{n})$.

${ }_{n} f_{x} \quad$ Fracción media de los últimos n-años vividos, siendo:

$$
{ }_{n} f_{x}={ }_{n} a_{x} / n
$$

Siendo ${ }_{n} a_{x}$ la media de años vividos por los ${ }_{n} d_{x}$ fallecidos en el rango de edad $[x, x+n)$, por ejemplo: 5 muertos en el rango de edad $[10,15)$, es decir de 10-14 años cumplidos $\left({ }_{5} d_{10}=5\right)$ cada uno de los cuales murió exactamente a la edad del siguiente esquema:

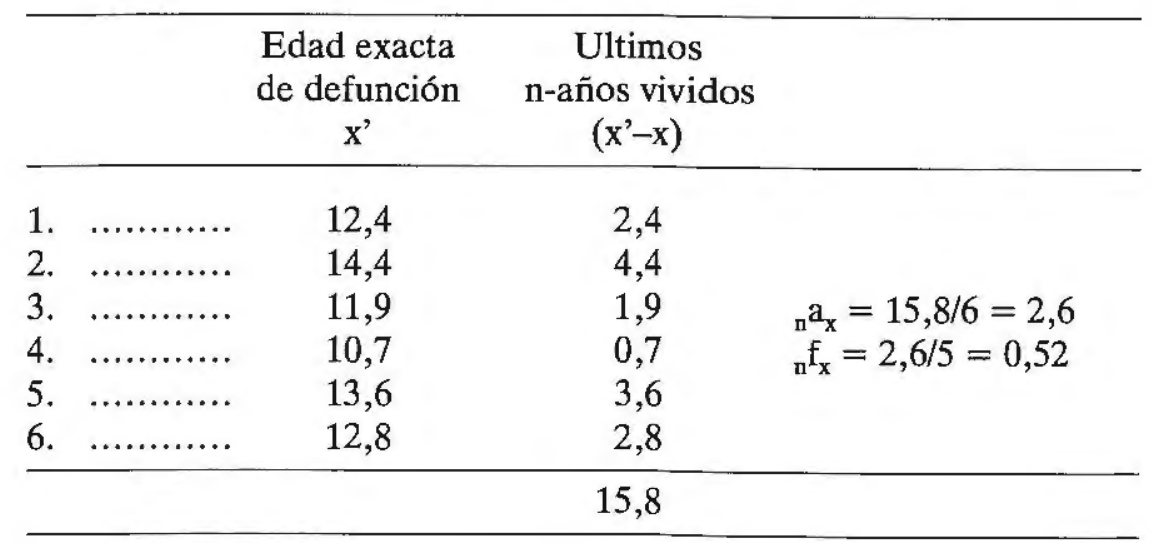


Para nuestras tablas de vida hemos elegido los valores de ${ }_{n} f_{x}$ estimados por Chiang desde un estudio empírico de una muestra de los certificados de defunción.

${ }_{n} \mathrm{~L}_{\mathrm{x}} \quad$ Número de años vividos por los $\mathrm{l}_{\mathrm{x}}$ supervivientes a $\mathrm{x}$, durante los siguientes $\mathrm{n}$-años. Los $\mathrm{l}_{\mathrm{x}+\mathrm{n}}$ supervivientes al final de período han vivido un total de $\mathrm{n}^{*} 1_{\mathrm{x}+\mathrm{n}}$ años, y los ${ }_{\mathrm{n}} \mathrm{d}_{\mathrm{x}}$ que murieron durante ese período habrán vivido ${ }_{n} a_{x}{ }_{n}{ }_{n} d_{x}$, es decir la media de años vividos por los que murieron en ese período (rango de edad) por el total de individuos muertos en el período. La suma de los años vividos por los que sobrevivieron al final de período $\left(1_{x+n}=1_{x}-{ }_{n} d_{x}\right)$ con los años vividos en el período por los que fallecieron en el $\left({ }_{n} d_{x}\right)$ nos da el total de años vividos en el período por $\operatorname{los}_{n} l_{x}$ iniciales. (Este es el concepto de Personas-tiempo). La fórmula general para su cálculo:

$$
{ }_{n} L_{x}=n^{*}\left[1_{n}-\left(1-{ }_{n} f_{x}\right) *{ }_{n} d_{x}\right]
$$

Para todos los grupos de edad menos para el último, sobre el que aplicando un modelo exponencial de fuerza de mortalidad constante, es estimada, dividiendo el Resto de defunciones (o superviviente) por la tasa de mortalidad de este último grupo de edad.

Los cálculos para la estimación de los diferentes parámetros de las tablas de vida se realizan de una manera seriada, tras la asunción general:

$$
{ }_{n} M_{x}={ }_{n} m_{x}
$$

$\mathrm{O}$ al menos es la mejor estimación que disponemos de $_{\mathrm{n}} \mathrm{m}_{\mathrm{x}}$. A partir de aquí:

1. Estimación de ${ }_{n} q_{x}$ :

$$
{ }_{n} q_{x}=\frac{{ }_{n} M_{x}}{(1 / n)^{*}\left[1+n^{*}\left(1-{ }_{n} f_{x}\right)^{*}{ }_{n} M_{x}\right]}
$$

2. Tomando una cohorte inicial de $100.000\left(1_{\mathrm{o}}\right)$ y aplicándole los riegos previamente estimados, realizar los diferentes cálculos seriados de supervivientes en cada edad:

$$
1_{x+n}=1_{x}^{*}\left(1-{ }_{n} q_{x}\right)
$$


3. Lo mismo para las defunciones a cada edad:

$$
{ }_{\mathrm{n}} \mathrm{d}_{\mathrm{x}}=\mathrm{1}_{\mathrm{x}}-\mathrm{l}_{\mathrm{x}+\mathrm{n}}=\mathrm{l}_{\mathrm{x}}{ }^{*}{ }_{\mathrm{n}} \mathrm{q}_{\mathrm{x}}
$$

4. Los años vividos en el período $\left({ }_{n} L_{x}\right)$, por los efectivos supervivientes $\left(l_{x}\right)$ al comienzo del período. Y el sumatorio de estos hasta la edad « $\mathrm{X}$ » o total de años vividos a $x\left(T_{x}\right)$ por el efectivo inicial $\left(l_{0}\right)$ de la generación:

$$
\mathrm{T}_{\mathrm{x}}=\text { Sumatorio }\left({ }_{\mathrm{g}} \mathrm{L}_{\mathrm{x}}\right) / \text { desde } \mathrm{i}=0 \text { hasta } \mathrm{x}=\mathrm{i}
$$

5. La esperanza de vida ax: por $e_{x}=T_{x} / l_{x}$.

\section{BIBLIOGRAFIA}

ALMOGUERA SALLENT, P.: «Estructuras demográficas andaluzas, 1981», Revista de Estudios Andaluces, n. ${ }^{\circ} 7,1986$, pp. 77-100.

CONSEJERIA DE POLITICA TERRITORIAL: Evolución reciente de la población y previsiones. Area metropolitana de Sevilla, Junta de Andalucía, 1985.

CONSEJERIA DE SALUD. Indicadores demográficos. Andalucta 1950-1981. Junta de Andalucía, Sevilla, 1984.

COZAR VALERO, E.: «La población andaluza. Movimiento natural y migraciones». En Geografia de Andalucia, (Director G. Cano), vol, III. Tartessos, Sevilla, 1987, pp. 15-28.

ELANDT-JHONSON, R.C.: «Survivat models and data analysis». Cap. IV. Life tables: fundamentals and construction. Wiley and Song, N.Y., 1978.

WORLD HEALTH ORGANIZATION: Manuel of Mortality analysis, W.H.O., Geneva, 1980.

PRESSAT, R.: El Análisis demografico. Fondo de Cultura Económica. México, 1967.

PUYOL ANTOLIN, B. «La población». En Geografía Humana. Cátedra, Madrid, 1988. 